Activités

16-1 | 2019

Comprendre le travail dans les "métiers adressés à autrui"

\title{
Affect et comparaison dans le dialogue en autoconfrontation
}

Affect and comparison in self-confrontation method

\section{Antoine Bonnemain}

\section{(2) OpenEdition}

\section{Journals}

Édition électronique

URL : http://journals.openedition.org/activites/4007

DOI : $10.4000 /$ activites.4007

ISSN : 1765-2723

Éditeur

ARPACT - Association Recherches et Pratiques sur les ACTivités

Référence électronique

Antoine Bonnemain, "Affect et comparaison dans le dialogue en autoconfrontation », Activités [En

ligne], 16-1 | 2019, mis en ligne le 15 avril 2019, consulté le 08 août 2019. URL : http://

journals.openedition.org/activites/4007; DOI : 10.4000/activites.4007

Ce document a été généré automatiquement le 8 août 2019

\section{c) $(7) \Theta$}

Activités est mis à disposition selon les termes de la licence Creative Commons Attribution - Pas d'Utilisation Commerciale - Pas de Modification 4.0 International. 


\title{
Affect et comparaison dans le dialogue en autoconfrontation
}

\author{
Affect and comparison in self-confrontation method
}

\section{Antoine Bonnemain}

\section{NOTE DE L'ÉDITEUR}

Article soumis le 8 février 2018, accepté le 21 décembre 2018

\section{Introduction}

1 L'analyse de leurs activités par les professionnels en autoconfrontation peut être regardée du point de vue du développement de leur activité pratique : le dialogue entre pairs a-t-il ou non donné lieu à des modifications concrètes de l'activité de travail ? Cette question est capitale, car elle pose le problème des méthodes d'action et des méthodes de recherche. En clinique de l'activité, l'autoconfrontation est d'abord une méthode d'action avant de devenir ensuite une méthode de recherche. Elle doit d'abord permettre d'organiser un développement du dialogue entre les professionnels d'un milieu, afin que ce développement et son devenir dans l'activité ordinaire de travail fassent ensuite l'objet de la recherche. Le chercheur tente alors de comprendre les mécanismes de ce développement réalisé pour enrichir en retour la méthode d'action, et pour organiser ensuite des cadres méthodologiques réglés qui tiennent compte des connaissances acquises vis-à-vis des organisateurs du développement de l'activité.

Dans cet article, nous proposons de regarder la méthode en autoconfrontation (Clot, Faïta, Fernandez, \& Scheller, 2001) comme un processus d'apprentissage. Les professionnels qui s'y engagent doivent en effet, nous y reviendrons ensuite, s'approprier le genre d'activité particulier du dialogue, incarné d'abord par le chercheur. C'est en effet ce dernier qui, explicitement ou implicitement, fixe la tâche à réaliser et les règles à suivre à des professionnels qui ne savent pas toujours spontanément ce qu'on attend 
d'eux. On peut du coup regarder l'autoconfrontation comme un processus d'apprentissage, à condition bien sûr de ne pas réduire l'apprentissage à l'intériorisation de connaissances nouvelles, mais de le définir plutôt comme l'appropriation d'un genre d'activités (Yvon, \& Clot, 2003). Le cadre proposé a des effets formatifs (Thébault, 2013) qui constituent l'une des conditions du développement de l'activité pratique. C'est ce processus que nous chercherons à instruire ici en suivant l'appropriation par un professionnel d'un artefact spécifique - la comparaison entre activités - et ses effets sur le développement de l'activité pratique et sur l'intervention en général. Nous verrons que l'affectivité y joue un rôle moteur.

3 Nous reviendrons d'abord sur ces questions en présentant les présupposés de la méthode du point de vue de la clinique de l'activité, depuis les travaux de Vygotski et Bakhtine, en lien avec la comparaison. Nous reviendrons ensuite sur la fonction développementale de la comparaison, qui est l'une des modalités qui organise les dialogues entre les professionnels dans l'intervention en clinique de l'activité. On instruira enfin le processus d'appropriation de cette modalité dialogique à l'aide de matériaux empiriques issus d'une intervention en cours auprès des éboueurs de la Ville de Lille, qui prend pour objet la réorganisation du service de propreté municipal.

\section{La méthode en autoconfrontation : quelques repères}

\subsection{Une clinique de l'activité pour transformer le travail et son organisation}

Disons-le d'abord de façon condensée : l'activité réalisée par un professionnel n'a pas le monopole du réel de l'activité. Ce qui se fait - et que l'on peut considérer comme l'activité réalisée - n'est jamais que l'actualisation d'une des activités réalisables dans la situation où elle voit le jour. Chez Vygotski, «l'homme est plein à chaque minute de possibilités non réalisées » (Vygotski, 2003, p. 76).

Entendue de cette manière, l'activité réalisée n'est qu'une composante de l'activité. Elle ne la résume pas. Ce qui ne s'est pas fait, mais qui aurait pu se faire, fait également partie de l'activité. Le réel de l'activité concerne ce qui ne se fait pas, mais aussi ce qu'on ne fait plus, ce qu'on cherche à faire sans y parvenir - le drame des échecs - ce qu'on aurait voulu ou pu faire, ce qu'on pense pouvoir faire ailleurs. L'activité possède donc un volume qui révèle l'existence de conflits « normaux » dans le cours ordinaire de la réalisation du travail. Il s'agit de conflits qui ne se réalisent pas seulement entre les individus, mais qui prennent leur source à l'intérieur de toute activité, et qui traversent chaque sujet. Leplat (2014) a bien vu cette conflictualité des critères inhérente à l'activité : « il y a conflit de critères, écrit-il, lorsque l'amélioration de l'estimation d'un critère conduit à la dégradation de l'estimation de l'autre» (p.22). On peut citer comme exemple le conflit bien connu en ergonomie entre le critère de rapidité et le critère de précision: «il est apparu qu'il était impossible de faire en même temps très vite et très bien » (Ibid.). Toute activité se mesure à ce type de conflits de critères pour lesquels on parvient, ou non, à trouver des issues acceptables. Mais justement, la possibilité de trouver des issues acceptables à ces conflits ordinaires de l'activité a à voir avec le développement du pouvoir d'agir qui définit la santé dans une perspective développementale. Cette 
possibilité est liée à la fois à la qualité du dialogue entre collègues qui permet de développer de nouvelles ressources pour arbitrer ces critères dans l'activité de chacun, mais aussi à la qualité du dialogue à construire avec la hiérarchie, qui décide de la conception des tâches et de l'organisation. L'activité pratique en conflit peut s'enrichir de ce type d'échanges.

La méthodologie d'intervention en milieu professionnel en clinique de l'activité tente de tenir compte de ces éléments pour engager des transformations dans le travail et dans l'organisation (Bonnefond, 2017; Massot, \& Simonet, 2017), afin que cette dernière puisse constituer une ressource pour santé et pour l'efficacité. C'est aussi l'objectif d'un certain nombre de travaux en ergonomie (Barcellini, 2017 ; Carta \& Falzon, 2017 ; Falzon, 2013 ; Rocha, Mollo, \& Daniellou, 2017). En clinique de l'activité, la méthode mobilisée permet aux sujets de dire quelque chose de ce qu'ils ont fait, afin qu'ils soient aussi en mesure de dire quelque chose de ce qu'ils pourraient faire, et même de faire par la suite quelque chose de ce qu'ils ont dit. Car c'est dans cet aller-retour organisé méthodologiquement entre ce que le sujet fait, ce qu'il ne parvient pas à faire et ce qu'il pourrait faire d'autre que peuvent s'élaborer, individuellement et collectivement, de nouvelles manières d'arbitrer les conflits de critères dans l'activité, élargissant ainsi le rayon d'action du sujet sur son milieu et sur lui-même.

7 Pour comprendre ces mécanismes de développement du pouvoir d'agir, il faut selon nous transformer les situations en organisant des «expériences de développement». Concrètement, cette expérience consiste à organiser la répétition de l'expérience vécue des sujets - au moyen de l'enregistrement vidéo notamment, car c'est ce «redoublement» qui permet potentiellement au sujet de découvrir de nouvelles possibilités d'action concrète. Il s'agit d'organiser la reprise des expériences vécues du sujet afin qu'il puisse « les avoir à sa disposition à titre d'objet pour d'autres expériences vécues» (Vygotski, 2003, p. 78). Ces découvertes peuvent déboucher sur un développement de son pouvoir d'agir et sur une extension de son rayon d'action, si important pour la santé (Canguilhem, 2002 ; Clot, 2008).

8 Mais ces découvertes sont aussi directement indexées, dans l'intervention en clinique de l'activité, à la possibilité pour le sujet de faire quelque chose du cadre méthodologique qui lui est proposé, c'est à dire de faire sien les attendus propres au genre de dialogue que nous cherchons à installer dans le milieu professionnel. Parmi ces attendus, la comparaison est de première importance. Dans l'autoconfrontation, il ne s'agit pas seulement d'adopter une posture réflexive sur l'activité de travail, mais aussi de discuter - au sens fort du terme - sa propre activité, puis celle de son collègue, pour les comparer. La fonction du chercheur consiste alors à soutenir cette activité de comparaison difficile pour celui qui s'y livre. Ce processus vise à interférer avec les fonctionnements habituels, c'est pourquoi le principe de comparaison a à voir avec l'affect et les émotions, nous y reviendrons ensuite. Avant de présenter à partir d'un exemple une forme d'appropriation concrète de la comparaison, on reviendra ci-dessous sur le dialogue et son fonctionnement dans l'autoconfrontation.

\subsection{Au-delà du déjà-dit : le dialogue dans l'autoconfrontation}

9 La méthode en autoconfrontation consiste à demander à des professionnels différents de commenter les images vidéo de l'activité d'un collègue en autoconfrontation croisée avant que celui-ci ne commente les siennes (Duboscq, \& Clot, 2010)ํ․ Auparavant, chacun 
des professionnels impliqués dans ce dialogue a réalisé avec le chercheur une autoconfrontation simple face aux images de son activité. Ces confrontations répétées préparent leur engagement réciproque dans des dialogues professionnels sur les manières de travailler, au sein d'un collectif de pairs dans un premier temps, puis avec la hiérarchie ensuite.

En clinique de l'activité, l'autoconfrontation, simple puis croisée, est d'abord conçue comme un « espace-temps différent » (Clot, \& Faïta, 2000) dans lequel le cadre dialogique favorise l'élaboration de la pensée à partir de l'expérience professionnelle. Dans cette perspective, la distinction méthodologique entre le réel de l'activité et l'activité réalisée vaut aussi pour l'activité dialogique d'analyse dans laquelle sont placés les professionnels.

11 Chez Bakhtine, le nœud de l'approche dialogique est constitué par les relations entre le dialogue intérieur et extérieur : " dans le dialogue, les répliques de l'un empiètent sur les répliques du dialogue intérieur de l'autre » $(1970$, p. 347). Dans l'échange « se heurtent et discutent non pas deux voix entières et monologiques, mais deux voix déchirées » et « les répliques ouvertes de l'une répondent aux répliques cachées de l'autre » (Ibid., p. 350). Pas de discorde entre les sujets ou de dissonances entre leurs voix qui ne soient aussi, simultanément, « interférence de deux voix à l'intérieur d'une seule » (Ibid., p. 355). Pour Bakhtine, ces dissonances sont souvent subtiles. Elles ne laissent que des traces fugitives dans l'énoncé et sont repérables surtout « dans les silences que ne justifient pas le sens de son discours, dans des changements de ton inexplicables par rapport à sa première voix, dans un rire déplacé, etc.» (1984, p. 354). En milieu de travail, nous faisons de ce plurilinguisme un moyen de développer le dialogue entre les professionnels en organisant précisément la comparaison entre leurs activités réalisées.

Pour Bakhtine, non seulement le dialogue est possible quand les interlocuteurs ne partagent pas les mêmes significations, mais c'est même la condition de son développement. Ce que nous partageons déjà n'est pas aussi intéressant que ce que nous ne partageons pas encore (Bender, 1998, p. 193).

Ce qui compte, c'est le développement possible qui sépare celui qui parle ou agit de ses fonctionnements habituels. C'est la motricité de l'inaccompli qui engage les attendus de la parole dans ce qui lui échappe encore.

14 L'autoconfrontation met au travail chez les professionnels ce volume du dialogue. Il permet, à partir du dialogue réalisé, de mettre au travail chez nos interlocuteurs ce qu'ils auraient pu dire de différent de ce qu'ils peuvent dire habituellement. En cherchant à rendre visible, dicible, pensable le fait que le dialogue réalisé n'est qu'une manière de dire parmi d'autres, nous cherchons à favoriser le développement de la pensée dans l'objectif de seconder le collectif dans le développement de son pouvoir d'agir sur son milieu et sur lui-même (Clot, \& Faïta, 2000). C'est ce cadre dialogique qui caractérise en partie l'expérience de développement citée plus haut : en mettant au travail la pluralité des voix au sein de collectifs de travail, la méthodologie vise à défaire et à dénouer les manières habituelles de dire le travail, afin de faire apparaitre de nouvelles manières d'envisager la situation, et afin que d'autres arbitrages deviennent possibles dans l'activité de chacun. C'est la condition pour que de nouvelles solutions pratiques jusque-là «inimaginables » puissent s'élaborer au sein du milieu professionnel face aux problèmes réels qui se posent dans le travail. 


\subsection{Comparaison et développement} essentielle dans le développement du dialogue, et par suite dans le développement de l'activité pratique. Elle constitue une condition pour faire apparaitre et rendre discutable les différents arbitrages réalisés dans une même situation de travail entre deux professionnels. Elle permet de distinguer ce qui est commun entre les manières de faire et qui relève ainsi du genre professionnel partagé - et ce qui est approprié par chaque professionnel et qui relève donc plutôt du style propre à chacun (Clot, 2008). C'est dans un processus de comparaison que le sujet peut éventuellement découvrir que l'évaluation qu'il porte sur son propre geste diffère de l'évaluation de son collègue sur ce même geste. En comparant leurs gestes et leurs actions dans une situation similaire, en permettant au professionnel de s'approprier quelque chose du geste de l'autre dans son geste propre, l'autoconfrontation est une méthode qui organise alors une voie artificielle de circulation et de comparaison des différentes manières de faire la même chose dans le milieu de travail.

fonction de la comparaison au principe de la méthode est donc d'abord d'organiser cette circulation des manières de faire et de dire entre les professionnels d'un milieu, dans l'espace-temps artificiel de l'autoconfrontation. C'est sa première fonction. Lorsqu'elle est appropriée par les professionnels qui en font l'instrument de leurs échanges, elle constitue le moyen de développer la fonction psychologique du collectif de travail comme ressource pour l'activité individuelle. La comparaison entre activités qui engage la circulation entre les différentes manières de faire la même chose permet potentiellement à chacun de faire quelque chose de l'activité d'autrui dans son activité propre. Elle développe les potentialités de l'action concrète de chacun en situation par la médiation du dialogue.

Il n'y a pas ici de contradiction ni d'opposition entre l'individuel et le social, mais un rapport de continuité : «le développement ne se fait pas vers la socialisation, mais vers l'individualisation des fonctions sociales" (Vygotski, 2014, p. 551). C'est ce processus d'individualisation $\mathrm{du}$ social en-soi qui structure toute la théorie vygotskienne du développement. C'est même la loi fondamentale du développement qu'il a résumé de la manière suivante :

«Toute fonction psychologique supérieure a été extérieure - ce qui signifie qu'elle a été sociale; avant d'être une fonction, elle a été relation sociale entre deux personnes. Les moyens d'agir sur soi ont été initialement moyens d'agir sur les autres, et des autres sur la personne. » (Ibid., p. 545).

Ce processus développemental se traduit par l'apparition systématique des fonctions à deux reprises, sur deux plans : «d'abord sur le plan social, puis psychologique, d'abord entre individus comme catégorie interpsychique, puis au dedans de l'enfant, comme catégorie intrapsychique» (Ibid., p. 286). D'abord source de l'activité individuelle, la situation sociale devient ressource pour cette activité individuelle.

Les dialogues entre pairs que la méthode des autoconfrontations organise visent précisément à produire le passage des ressources sociales extérieures à l'intérieur de chacun, comme ressources au service de l'activité individuelle (Fäita, 1999, 2011; Kostulski, 2004; Kostulski \& Clot, 2007). C'est à la condition d'une sorte de collision interne répétée qui prend d'abord sa source dans la vie sociale et collective que le sujet pourra choisir entre différentes possibilités qu'il porte alors en lui pour agir dans la 
situation, qu'il pourra donc étendre son rayon d'action. Mais c'est aussi seulement à cette condition qu'il pourra en retour retoucher ces ressources sociales et les rendre éventuellement disponibles pour d'autres. C'est là la fonction psychologique principale d'un collectif de travail dynamogène.

Comme dans l'activité pratique, le fonctionnement de l'activité dialogique en autoconfrontation est conflictuel (Kostulski, \& Clot, 2007). Les interlocuteurs ne partagent pas toujours les mêmes significations. C'est d'ailleurs la source de son développement: il ne peut advenir de nouveau dans l'échange que lorsque les professionnels sont placés dans un cadre qui ne renie pas cette structure conflictuelle, mais qui en fait au contraire un moyen d'aller au-delà des évaluations habituelles, du « déjà-dit ». C'est le point de départ potentiel d'un nouveau devenir de la pensée dans le mot, c'est à dire d'une pensée qui reconfigure ensuite le rapport à l'action en situation de travail. C'est pourquoi, dans le cours des autoconfrontations, le chercheur tente de soutenir l'échange autour des différentes manières de faire lorsqu'il se présente, car il constitue un indice de cette conflictualité en cours, et donc un indice de développements potentiels à venir.

Dans l'échange en autoconfrontation émergent fréquemment des désaccords entre collègues sur les manières de faire et sur les manières de dire. Ces désaccords, qui prennent leur source dans la comparaison, peuvent constituer le moyen de nouveaux développements de la pensée des sujets. L'affectivité joue dans ce processus un rôle central que nous allons maintenant caractériser. La comparaison endosse alors une seconde fonction, affective cette fois, tout aussi indispensable pour le développement de l'activité.

\subsection{Comparaison et affectivité}

$21 \mathrm{Au}$ sein du processus de comparaison, les « disputes professionnelles » qui se réalisent parfois au cours des autoconfrontations constituent, dans le cadre de la méthode, «l'énergie» d'une « migration fonctionnelle» (Kostulski, \& Clot, 2007; Vygotski, 2003) des ressources sociales dans l'activité individuelle. Ce que nous nommons ici «énergie » doit être rapproché de la question des affects et des émotions qui connait depuis quelques années un regain d'intérêt dans le champ du travail, soit pour comprendre leur fonctionnement propre au sein d'un «travail émotionnel» (Hochschild, 2003) potentiellement coûteux pour la santé (Ribert-Van de Weerdt, 2011), soit pour mieux comprendre leur motricité dans l'intervention (Bonnefond \& Clot, 2016).

Dans cette dernière perspective, le processus de «migration fonctionnelle » ne peut se réaliser que par la médiation d'une relation sociale spécifique - d'un type de dialogue particulier - dans laquelle la comparaison est source d'affects (Veresov, 2014). C'est la seconde fonction de la comparaison dans le dialogue en autoconfrontation. La comparaison produit de l'affect dans le dialogue, et l'affectivité joue un rôle moteur dans le développement de l'activité (Clot, 2017).

23 Si la « dispute » entre les opérateurs est motrice pour le développement du dialogue et de la pensée, c'est parce qu'elle est profondément affective. La comparaison entre activités engage les professionnels dans une expérience affective qui peut « décontenancer» les antécédents de leur propre discours (Bonnemain, 2015 ; Poussin, 2014). Ce qu'on pensait jusque-là justifié, ce qui était incorporé, ce qui organisait jusque-là l'activité, peut se trouver désorganisé par la discussion en cours, pris en défaut dans l'inattendu des 
échanges. Ainsi, la modification du point de vue des professionnels sur les problèmes qui se posent à eux dans leur activité pratique est reliée de l'intérieur à la possibilité pour eux d'être affectés par la nouveauté, parfois inattendue, qui surgit dans l'échange.

C'est le sens de la comparaison recherchée par la méthode : la comparaison constitue une condition importante du développement des disputes professionnelles, qui peuvent à leur tour produire une énergie suffisante pour la migration des ressources extérieures dans l'activité propre d'un sujet. La comparaison, conçue ici d'abord comme artefact - objet d'appropriation possible - dans l'autoconfrontation, peut se muer en instrument du dialogue et engage alors des mouvements affectifs dans l'activité des professionnels dans l'autoconfrontation. On soutiendra en ce sens dans l'exemple clinique qui suit que la comparaison met «en activité » l'affectivité des sujets - ce système fonctionnel et dynamique des affects, des émotions et des sentiments (Bonnefond \& Clot, 2016) jusqu'au développement éventuel de leur activité pratique.

Dans l'autoconfrontation, il faut alors aussi regarder le développement du dialogue du côté des affects qui se réalisent dans la situation de l'échange verbal. Cet échange et son développement ne peuvent se concevoir seulement comme une transformation linéaire et « désaffectée » de la parole. Au contraire, Bakhtine annonçait déjà la fonction des affects et des émotions dans ce processus développemental: dans le dialogue "l'activité de l'auteur devient l'activité d'une évaluation exprimée qui colore tous les côtés du mot: le mot invective, caresse, exprime son indifférence, rabaisse, délivre, etc. » (Bakhtine, 1924, cité par Peytard, 1990, p. 11). Le mot véhicule l'affect, il le trahit en laissant transparaître les émotions auxquelles il s'associe en situation.

L'échange engage donc toujours une évaluation teintée d'affectivité. Dans le dialogue «le mot ne désigne pas seulement l'objet comme une certaine entité disponible, mais exprime aussi mon attitude évaluative par rapport à cet objet» (Bakhtine, 2003, p. 58). Cette évaluation s'appuie sur un conflit inéliminable qui se réalise toujours dans le dialogue entre les attendus du sujet et les inattendus qui surgissent dans la situation de l'échange verbal : découvrir par exemple que son collègue voit la même situation ou le même geste autrement que soi-même. Ainsi, la possibilité de modifier ses façons de faire ne va pas de soi, elle suppose la création d'une énergie affective à même de supporter la reprise et la transformation de l'expérience « déjà-vécue », jusque dans le mot dans laquelle elle prend forme.

27 L'affect est ce devenir de l'activité qui met en balance les attendus et les inattendus. Il produit une énergie pour l'action sans garantir sa réalisation effective, en provoquant soit un «blocage », soit une "révision » de l'action habituelle (Livet, 2007, p. 341). Il est un conflit plus ou moins intense entre «l'expérience qu'on a » et «l'expérience qu'on fait » en situation (Pastré, 2012, p. 120) qui modifie le régime de l'activité, soit du côté des défenses et de la passivité, soit du côté d'une transformation motrice de l'activité. L'affect est cette oscillation permanente et jamais définitive entre des formes de passivité qui tentent de rabattre l'inattendu sur ce qu'on connaît déjà, et des formes d'activité qui font de l'inattendu le moyen de faire une expérience nouvelle et inédite (Bonnemain, 2015).

Il faut alors mesurer à quel point la situation d'autoconfrontation comporte des contraintes et des exigences pour les sujets qui s'y engagent dans le cours de l'intervention, la nécessité de comparer son activité à celle d'autrui en est un exemple. Il s'agit d'une situation de réflexivité outillée par un certain nombre de moyens prédéfinis, artefacts disponibles pour le dialogue à conduire entre collègues, et avec le chercheur. Or, l'appropriation de ces artefacts, condition d'un dialogue qui favorise le dépassement du 
déjà-dit, ne va pas de soi. C'est pourquoi elle doit faire l'objet d'une attention clinique particulière de la part du chercheur. Car « pour qu'une situation entre dans le champ de l'activité d'une personne, celle-ci est conduite à s'approprier aux situations, autrement dit, à s'outiller des règles, des outils, des fonctions et des signes qui permettent d'en faire l'expérience et pas seulement d'en avoir l'expérience» (Mayen, 2012, p. 305). Nous présenterons donc ensuite un exemple d'appropriation de la comparaison en situation d'autoconfrontation par un professionnel éboueur engagé au sein d'un dispositif d'intervention. Au cours de ce travail, il fait de la comparaison un moyen de l'échange avec ses collègues. C'est pour nous la trace conservée d'un apprentissage en cours de réalisation : l'apprentissage d'un genre de dialogue spécifique, qui fait de la comparaison entre activités le moyen de repenser autrement un objet ou un problème particulier en affectant au passage l'activité d'autrui et la sienne propre. C'est de cette forme de genèse instrumentale dont il s'agira ensuite.

Nous reviendrons donc maintenant sur l'analyse de l'appropriation de ce genre de dialogue dans l'autoconfrontation au sein d'une intervention en cours auprès des éboueurs de la Ville de Lille.

\section{Un exemple chez les éboueurs de la Ville de Lille : la comparaison comme instrument du dialogue}

\subsection{L'intervention et son contexte}

30 Pour présenter les données que nous mobilisons ensuite, nous revenons ici sur l'origine et le déroulement de l'intervention. La commande d'intervention nous est adressée par la directrice des ressources humaines de la Ville et le directeur du service de propreté. La directrice des ressources humaines souhaite que cette action puisse permettre d'expérimenter dans l'organisation une autre manière de réorganiser les services opérationnels. En effet, elle constate que les réorganisations sont habituellement menées " par le haut ", c'est-à-dire sans véritable participation des opérateurs de première ligne. Les décisions sont la plupart du temps prises sans eux, et la direction cherche un moyen pour définir les contours d'une participation réelle des salariés aux réorganisations du travail. Le directeur du service de propreté est engagé dans cette commande par la médiation de la directrice des ressources humaines. Le service est au centre de multiples enjeux municipaux importants et doit faire l'objet d'une réorganisation qui vise l'amélioration de la performance des prestations de propreté. Le directeur voit dans cette action une opportunité pour être " accompagné » par des psychologues du travail afin de penser cette réorganisation « avec » les professionnels de terrain, et non pas « pour » eux. Le service compte environ 130 éboueurs répartis au sein de 6 équipes territorialisées sur l'agglomération et les communes associées.

31 C'est sur la base de cette commande que nous avons mis en place, en concertation avec les décideurs, un dispositif d'intervention à trois niveaux dans le but de développer performance et santé en instituant dans l'organisation le conflit de critères sur la qualité du travail :

1. Un comité de pilotage réunissant les principaux dirigeants de l'organisation, la direction du service et les intervenants psychologues du travail ;

2. Un comité de suivi réunissant la direction, les organisations syndicales et les intervenants; 
3. Un collectif de professionnels éboueurs, en charge de faire l'analyse de leurs activités de travail en autoconfrontation, afin d'en discuter ensuite avec la hiérarchie pour trouver des réponses concrètes aux problèmes qui s'y posent.

32 L'intervention a débuté en octobre 2016 et dure maintenant depuis plus d'un an. Sans entrer davantage dans le détail de l'intervention qui ne constitue pas l'objet de cet article, on peut néanmoins en rappeler les trois principales phases méthodologiques (Bonnemain, Bonnefond, Fontes, \& Clot, sous presse).

La première phase a duré quatre mois. Elle visait à organiser le développement de la fonction psychologique du collectif de travail au sens où nous l'avons définie plus haut. C'est le temps des observations du travail réel et des autoconfrontations simples et croisées auprès des professionnels concernés. 14 autoconfrontations simples et 7 autoconfrontations croisées ont été réalisées auprès des éboueurs d'une des six équipes que compte le service. 14 éboueurs ont accepté de s'engager dans ces discussions entre eux sur leur activité.

Ce travail a produit deux résultats : d'abord, les échanges entre éboueurs ont permis de développer les ressources sociales au service de l'activité individuelle. On verra ensuite à partir de notre exemple clinique comment le dialogue peut produire de nouvelles ressources pour l'action des uns et des autres en modifiant éventuellement les manières de faire le travail. Ensuite, cette phase permet aux éboueurs de réaliser un certain nombre de diagnostics sur les situations qui font problèmes et qui mériteraient d'être discutées avec la hiérarchie pour y trouver des réponses concrètes.

La seconde phase organise le développement de la fonction sociale de ce même collectif, dans l'organisation cette fois. Elle vise à organiser l'échange répété entre des éboueurs et la hiérarchie jusqu'au plus haut niveau de l'organisation. De ce point de vue, 7 comités de pilotages ont été réalisés, dont 6 en présence des éboueurs ou de ce qui a été désigné ensuite comme des "référents-métier ", mandatés par leurs collègues pour représenter le travail réel auprès de la hiérarchie. D'autre part, au cours de cette phase, une nouvelle instance de traitement des problèmes-opérateurs a été mise en place au niveau de la direction de la propreté. Elle permet un dialogue régulier entre encadrement (direction, agents de maîtrise) et éboueurs " référents » pour organiser concrètement le traitement des problèmes liés de près ou de loin à la possibilité de faire un travail jugé de qualité par ceux qui le réalisent. Lors de cette phase, une dizaine de problèmes relevés par les référents-métier de l'équipe ont pu être traités en coopération avec la direction et l'encadrement intermédiaire.

La dernière phase organise la généralisation du dispositif à l'ensemble des équipes du service, sur la base de l'expérience faite jusque-là à l'échelle d'une seule équipe. Un référent-métier est désigné par ses collègues dans chaque équipe du service. Il doit répondre à une tâche précise pour recueillir et discuter les problèmes à résoudre avec ses collègues d'abord, puis avec la hiérarchie lorsque cela est nécessaire. C'est aussi la phase dans laquelle le rôle des organisations syndicales s'intensifie afin de garantir la continuité du dispositif dans le temps.

Ce dispositif, qui organise ce qui a été désigné ailleurs comme une "coopération conflictuelle » (Trentin, 2012) entre opérateurs, hiérarchies et organisations syndicales, a permis aujourd'hui de traiter une cinquantaine de problèmes divers (organisation, matériel, sécurité, etc.) relevés par les éboueurs comme des obstacles à la performance, à la santé ou à la sécurité. Mais le travail réalisé jusque-là est encore amené à se poursuivre 
et d'autres problèmes devraient encore être résolus dans la poursuite de l'action. L'enjeu ici est de pouvoir instituer ce genre de fonctionnement dans l'organisation de manière durable, en s'appuyant sur le développement du dialogue aux différents niveaux et entre les niveaux.

Mais pour comprendre ce développement du dialogue et les résultats obtenus que nous avons rapidement présentés ici, il faut revenir, comme nous allons le faire maintenant, sur la manière dont ce genre de dialogue a d'abord été approprié par les professionnels concernés entre eux. C'est dans «l'espace-temps » des autoconfrontations qu'ils ont pu s'approprier les artefacts dialogiques qui expliquent en partie les résultats obtenus ensuite. Dans ce type de dispositif, la " performance dialogique » entre les professionnels, puis entre eux et la hiérarchie, constitue une condition du développement de la performance tout court (Bonnefond, Bonnemain, Fontes, \& Clot, 2016). Entrer dans la comparaison entre activités constitue ici une condition de la performance dialogique, définie par la capacité du dialogue à produire quelque chose de neuf (Ibid.).

\subsection{Un exemple clinique : l'appropriation de la comparaison par la traversée des contextes}

Nous présenterons ici un exemple d'appropriation de la comparaison en autoconfrontation. Nous le ferons en suivant pas-à-pas la succession des dialogues réalisés par l'un des éboueurs, Christophe, qui a réalisé une autoconfrontation simple et deux autoconfrontation croisées, avant de jouer un rôle important ensuite dans l'intervention, dans l'échange avec la hiérarchie. On cherchera à illustrer la manière dont Christophe fait progressivement de la comparaison un instrument de dialogue avec ses collègues et comment cette appropriation produit des mouvements affectifs dans le dialogue. Cette appropriation se réalise dans ce cas, comme on le verra, dès l'autoconfrontation simple. Le film faisant l'objet de la discussion porte sur l'activité de balayage de Christophe que nous avons filmée, tâche sur laquelle il a été affecté la veille par son agent de maîtrise. Cette première situation en autoconfrontation simple est un premier espace dans lequel le professionnel s'approprie quelque chose de la méthode proposée.

\subsection{L'autoconfrontation simple : la comparaison comme préoccupation du chercheur}

Dans la séquence suivante, le chercheur a lancé la diffusion du film sur l'écran de l'ordinateur. Deux minutes complètes s'écoulent sans que Christophe ne dise rien de ce qu'il se voit faire sur ces images. La séquence débute au moment où le chercheur arrête le défilement des images afin de tenter d'engager davantage Christophe à s'expliquer avec la situation filmée.

Tableau 1 : Extrait de l'autoconfrontation simple réalisée avec Christophe. Table 1: Extract from self-confrontation with Christophe

1 Chercheur : (arrête le film) Donc tu vois moi ce que je cherche à comprendre c'est aussi... c'est les problèmes - tu vois hier on a discuté du temps d'attente et de trucs comme ça

2 Christophe : ouais 
3 Chercheur : mais c'est pas seulement ça c'est aussi les techniques tu vois. Parce que j'ai remarqué que vous faites pas- vous faites pas tous pareil hein. Tu vois toi comment tu tiens ton balai et ben Serge il le tient pas comme ça son balai.

4 Christophe : il est trop petit Serge...

5 Chercheur : ouais mais tu vois moi pour moi je... je comprends pas quoi... Je comprends pas pourquoi des fois il faut tirer, pourquoi des fois il faut pousser enfin on va regarder ça quoi, mais ça ça m'intéresse

6 Christophe : ah ouais d'accord

7 Chercheur : (relance le film)

8 Christophe : ah ouais donc toi tu veux savoir... donc là par exemple on est gêné avec les roues et tout euh... tu vois c'est chiant mais tu veux que je te dise quoi en fait?

9 Chercheur : euh... ben je sais pas là par exemple (pointe l'écran) là ça passe par exemple?

10 Christophe : ouais. Mais après ça passera pas. Je me rappelle regarde je crois que c'est là que ça passe pas. Tu vois hop.

11 Chercheur : oui, mais tu y arrives quand même.

12 Christophe : ouais mais regarde, il en reste.

13 Chercheur : ah ouais... et ça tu peux pas l'enlever c'est?

14 Christophe : ben non parce c'est... c'est chiant... tu veux faire comment?

15 Chercheur : ouais parce que sinon tu vas y passer du temps.

16 Christophe : bah même euh... tu veux faire comment avec les roues et tout c'est chiant. Tu vois c'est une question de circulation là tu vois... Je sais pas comment on peut faire...

17 Chercheur : ouais d'accord

18 Christophe : tu vois c'est pfff... c'est embêtant.

19 Chercheur : donc il y a des voitures qui sont bien garées et d'autres euh...

20 Christophe : ben ouais parce que après tu vois... tu vois quand tu traverses c'est bien de regarder à droite et à gauche tu vois...

21 Chercheur : attends je vais changer de lecteur de vidéo parce que là ça marche mal... (ferme le logiciel et en ouvre un autre)

22 Christophe : de toute façon ce qu'on se dit là pour le moment c'est pour nous ensuite on va couper et tout?

23 Chercheur : ouais c'est pour nous pour l'instant on verra ensuite. Mais après ce qu'on fera c'est que on va reprendre ces images-là et on en discutera avec euh soit Serge soit... un autre collègue qui fait la même chose.

24 Christophe : Fabrice il est plutôt au balai, Smaïn il est tout le temps au balai

25 Chercheur : je les ai pas filmés encore, mais...

26 Christophe : Fabrice, Smaïn...

27 Chercheur : il faut que je les filme aussi

28 Christophe : Smaïn. Souvent au balai lui... Euh... ouais nan après oui parce qu'après c'est bien aussi d'avoir d'autres avis comme tu dis tu vois

29 Chercheur : ah oui ah oui

30 Christophe : tu seras bien comme ça. Parce que... Après c'est bien d'avoir aussi une discussion avec un autre collègue

31 Chercheur : ben oui

32 Christophe : tu vois ? Comme ça nous on peut parler de...

33 Chercheur : de ce que vous connaissez quoi 
34 Christophe : de ce qu'on connaît et on peut se... contredire un peu.

35 Chercheur : ouais.

36 Christophe : parce que toi comme tu sais pas tu vois

37 Chercheur : ouais... mais c'est pour ça d'abord on en discute là et ensuite on fera ça.

38 Christophe : ben ouais. Parce que tu vois moi je vais dire « ben nan c'est mieux comme ça » et lui il va me dire « ben nan regarde » tu vois. Parce qu'on sait pas... nan, mais tu vois on sait pas comment... comment...

39 Chercheur : ouais.

40 Christophe : donc on pourra se confronter un petit peu

41 À la suite de cet extrait, se déploie un échange que nous ne pouvons rapporter ici entre le chercheur et Christophe sur la réalisation de son geste de balayage et sur la manière dont il pousse les déchets avec son balai.

Dans cet extrait, Christophe ne sait pas bien ce qui est attendu de lui dans le cadre de l'autoconfrontation simple. Au départ, l'observation des images filmées de son activité de balayage ne suscite chez lui aucune réaction extérieure. C'est pourquoi le chercheur arrête les images pour tenter d'expliquer à nouveau les objets potentiels de ce dialogue : les «problèmes» posés par l'activité pratique et les «techniques» employées pour balayer. Mais ce second objet est aussitôt associé par le chercheur aux différences entre les techniques employées par les éboueurs : «Serge il le tient pas comme ça son balai » (en 3). Cet énoncé introduit dans l'échange l'intérêt du chercheur pour la comparaison entre les différentes manières de faire la même chose chez les professionnels. Cet objet sera ensuite repris de manière indirecte par Christophe («après c'est bien aussi d'avoir d'autres avis comme tu dis ", en 28). La fin de l'extrait montre comment Christophe tente de faire quelque chose de cet intérêt pour la comparaison. Il envisage la possibilité pour lui de discuter avec certains collègues de ses images filmées (en 30), dans le but d'expliquer au chercheur les problèmes rencontrés et les techniques de travail mobilisées (en 36), et aussi sans doute dans le but d'aider le chercheur à recueillir les données dont il a besoin ("tu seras bien comme ça », en 30).

Cet extrait est pour nous capital pour comprendre comment peut s'engager l'appropriation par le professionnel de la comparaison, dans le cours des dialogues en autoconfrontation. Ici, c'est le chercheur qui fait d'abord de la comparaison un objet de préoccupation, sans que le mot même de «comparaison » ne soit thématisé. C'est en quoi le chercheur incarne en premier lieu les contraintes et les exigences du genre de dialogue à conduire entre les professionnels eux-mêmes. Il incarne l'orientation possible des dialogues sur la confrontation des manières de faire différentes pour atteindre les mêmes objectifs ou s'en fixer d'autres. C'est pour Christophe la première expérience du plurilinguisme professionnel dans l'autoconfrontation, en l'absence des collègues qui sont d'abord rendus présents par le chercheur lui-même (en 3). Ce faisant, la comparaison peut potentiellement devenir l'objet poursuivi également par le professionnel dans la suite de l'intervention, et notamment dans les autoconfrontations croisées à venir. La comparaison peut devenir, pour différentes raisons à retrouver dans chaque situation singulière, un moyen technique du dialogue avec les collègues. Notons qu'ici, la comparaison constitue un objet indirect de l'échange entre le chercheur et le professionnel. Elle constitue un artefact de la situation d'analyse de l'activité, disponible pour le professionnel et sollicitée par le chercheur qui l'induit - sans la nommer de 
manière explicite - par ses questionnements. C'est le devenir de la comparaison comme technique du dialogue que nous allons chercher à instruire dans la poursuite des échanges entre Christophe et ses collègues. Il s'agira d'instruire, à partir de cet exemple, la manière dont s'intériorise chez les professionnels le genre de dialogue spécifique décrit précédemment, qui vise à dépasser le déjà-dit et le déjà pensé pour qu'une autre compréhension de la situation soit possible, et du coup un développement éventuel de l'activité pratique dans d'autres gestes possibles. Nous verrons au cours de ce processus que la comparaison, lorsqu'elle devient l'instrument du dialogue, produit des mouvements affectifs moteurs pour le développement de l'activité.

\subsection{La première autoconfrontation croisée : la comparaison comme instrument du dialogue}

La première autoconfrontation croisée réalisée par Christophe est effectuée avec son collègue Serge. L'objectif consiste à produire des commentaires croisés de l'activité déjà discutée avec le chercheur dans l'autoconfrontation simple, cette fois-ci en présence d'un collègue qui s'est lui-même prêté à cette expérience. La séquence suivante montre comment Christophe fait quelque chose de la comparaison dès le début de la séance de travail avec son collègue.

Tableau 2: Extrait d'autoconfrontation croisée entre Christophe et Serge.

Table 2: Extract from crossed self-confrontation between Christophe and Serge

1 Chercheur : Donc... j'avais noté plusieurs choses qu'on avait commencé à discuter qui sont importantes pour moi... il y avait les gestes

2 Christophe : oui

3 Chercheur : la question des gestes on en avait parlé chacun ensemble

4 Christophe : oui la posture

5 Chercheur : euh... il y avait le problème des voitures. Les problèmes de voitures et de parking qui étaient embêtants. Euh... les techniques, pousser ou tirer les déchets au milieu de la route ou pas... voilà il y avait tout ça. Ça serait bien qu'on essaye de revenir un peu là-dessus et puis que vous puissiez essayer de regarder aussi précisément comment pour Christophe comment lui il s'y prend et pour Serge comment il s'y prend.

6 Christophe : (à Serge) ouais ça on en avait parlé ah ouais. On en avait parlé. Et voilà... pour ta taille en fait...

7 Serge : ben ouais

8 Chercheur : et en fait le but c'est aussi de voir les problèmes de travail que vous rencontrez, par exemple le problème des voitures c'est peut-être un problème important...

9 Serge : ah ouais

10 Chercheur : ... par rapport à ce que la direction voudrait réorganiser. On peut imaginer que ce problème-là par exemple vous puissiez en discuter avec eux ensuite pour voir comment il faudrait organiser le travail sur ce problème... On m'a dit qu'avant il y avait des opérations de nettoiement?

11 Christophe : ouais... ils banalisaient la rue complète et c'était pas mal ça

12 Chercheur : et maintenant ça n'existe plus ça?

13 Christophe : non 
14 Chercheur : voilà des choses comme ça quoi... Bon on peut commencer par le film de Serge et après on fera le film de Christophe (lance le film)... Donc Christophe tu peux arrêter les images quand tu veux, tu fais comme ça (lui montre sur l'ordinateur)

15 Christophe : ah ok et je commente sur Serge

16 Chercheur : ouais

17 Christophe : (regarde les images) tu veux que j’te dise quoi moi ? (à Serge)

18 Serge : rien (rire)

19 Christophe : ben Serge, une question (arrête le film). Pourquoi tu tiens ton balai comme ça ? Parce que l'autre fois il m'a demandé...

20 Serge : ben moi aussi il m'a demandé

21 Chercheur : comment, comment il le tient?

22 Christophe : pourquoi il le tient comme ça (montre sur les images)

23 Chercheur : avec la main comme ça tu veux dire?

24 Christophe : ouais

25 Serge : euh... c'est machinal c'est comme ça

26 Christophe : c'est comme ça, il y a pas de raison?

27 Serge : nan il y a pas de raison précise

28 Christophe : (au chercheur) tu vois il y a pas de raison, nan c'est parce que il le tient comme ça

29 Serge : nan parce que au boulot ou à la maison je le tiens pareil, je le tiens toujours au même endroit. Avec une pelle je la tiens par le haut et au milieu.

30 Chercheur : (à Christophe) ouais mais parce que toi tu... on avait discuté de ça parce toi tu...

31 Christophe : moi je tire ouais

32 Chercheur : voilà donc la question c'est pas pourquoi... c'est plutôt que je me dis que ça doit changer quelque chose de le tenir plutôt comme ça ou comme ça.

33 Serge : ben j'ai toujours, moi personnellement j'ai toujours tenu un balai comme ça, mais depuis des années. Donc je sais pas si ça a une importance

34 Chercheur : ça a une importance ou pas?

35 Christophe : ben moi je pourrais pas le tenir comme toi (regard surpris de Serge) parce que j'ai l'habitude de le tenir en dessous

36 Serge : ben ouais c'est parce que toi tu... toi t'as pas l'habitude comme ça moi j'ai l'habitude depuis, depuis combien d'années

37 Christophe : ben ouais

38 Chercheur : (à Christophe), mais ça change quoi toi pour toi quand tu le tiens en dessous comme ça?

39 Christophe : ben c'est parce que je le ramène (mime le geste de balayage)... Parce que comme ça je pourrais pas faire comme ça (mime l'alternative gestuelle à partir du geste de Serge)... ce geste-là

40 Serge : mais je le ramène aussi

41 Christophe : nan, mais je veux dire moi ce geste-là (mime le geste) je pourrais pas le faire... moi, enfin tu vois j'y arrive pas

Revenons sur le déroulé de cet extrait. Le chercheur commence par rappeler les objets qui avaient été discutés lors des autoconfrontations simples avec Christophe et Serge (en 1 et 5). Il induit également à nouveau l'importance de la comparaison comme moyen de la 
discussion («que vous puissiez essayer de regarder aussi comment pour Christophe comment lui il s'y prend et pour Serge comment il s'y prend », en 5).

Christophe est " naturalisée » : à suivre les deux professionnels, il n'y aurait pas de raison de tenir son balai plutôt comme Serge ou plutôt comme Christophe. Le geste incorporé est difficilement verbalisable, et les deux professionnels butent visiblement sur cet obstacle face aux questionnements du chercheur qui insiste (en 21, en 23, en 32). C'est pourquoi ces énoncés (en 28 et 29) peuvent également être regardés comme des formes de "déjà-dit » qui visent à rétablir la tranquillité habituelle que la comparaison vient perturber. En 33, Serge énonce explicitement son questionnement sur l'importance de s'interroger sur le geste («je sais pas si ça a une importance»). Dans cette situation, les deux éboueurs tentent donc, chacun leur tour, d'exprimer leur scepticisme quant à l'objet en cours de discussion introduit d'abord par le chercheur en autoconfrontation simple.

C'est, pour eux, un étonnement que d'être questionné et de se questionner sur le geste ordinaire de travail qui ne fait pas spontanément l'objet des échanges dans l'activité quotidienne. Mais c'est également un inconfort que de devoir rentrer sur cette question par la comparaison qui est induite régulièrement par les relances du chercheur (par exemple en 30 et en 32). C'est pourquoi toute l'activité du chercheur consiste ici à soutenir les efforts des éboueurs face aux difficultés à entrer dans la comparaison entre 
activités : difficulté à «dire " son geste d'abord, et difficulté à le comparer au geste d'autrui ensuite.

51 Les deux professionnels ne sont pas simplement ici en train d'expliciter leur geste. Ils se confrontent d'abord à l'obstacle d'un « difficile à dire » (François, 1998, p. 26) bien connu en analyse du travail, inconfort qu'ils s'efforcent d'abord de rediriger vers le chercheur en tentant d'invalider la pertinence même de l'objet en cours de construction dans l'échange (en 28).

52 En 34, plutôt que de répondre à Serge sur l'importance de l'analyse du geste, le chercheur retourne la question aux deux professionnels («ça a une importance ou pas? »). C'est une tentative pour ne pas imposer une discussion sur le geste qui produit visiblement un inconfort chez les deux éboueurs, une manière de les laisser décider de l'issue de la conversation par eux-mêmes.

Mais plutôt que d'invalider définitivement cet objet de discours, comme on aurait pu s'y attendre, l'énoncé de Christophe en 35 le poursuit à nouveau en comparant les deux gestes (« moi je pourrais pas le tenir comme toi »). Cet énoncé marque un engagement de Christophe dans la comparaison entre les deux gestes, il permet de statuer sur leur différence, ce qui n'avait pas été formulé de manière aussi explicite jusque-là. Dans cette seconde partie de l'extrait (de 35 à 41), la comparaison qui était d'abord évitée par les professionnels change de statut dans l'activité de Christophe, elle organise maintenant son activité langagière à la recherche des différences entre les deux gestes, comme en atteste la fin de l'extrait. Cette modification du régime de la comparaison est en effet repérable en 39 et en 41, lorsque Christophe mime le geste de son collègue, ou lorsque la comparaison se poursuit sur le fait de " ramener » le déchet avec son balai. En cherchant à expliquer à son collègue les raisons pour lesquelles il ne pourrait pas réaliser le même geste que lui, Christophe est poussé à rendre explicite - autant que possible - leur différence. C'est pourquoi il mime le geste de Serge pour rendre cette différence plus explicite. Ce faisant la comparaison ne sert plus seulement à construire un objet de discours possible comme c'était le cas au début de l'extrait, elle les engage à la fin de l'extrait à s'expliquer avec leurs gestes, éventuellement en mimant le geste du collègue, afin d'objectiver davantage ces différences.

54 Notons que le principe de comparaison n'est jamais explicitement nommé par les professionnels alors même que la seconde partie de l'extrait montre que la comparaison est à l'œuvre entre eux. En effet, dans cette situation, la comparaison ne fonctionne pas selon nous comme un concept verbalisable par les professionnels ni comme un objet de connaissance formalisable, mais agit plutôt comme un "concept pragmatique" (Samurçay \& Pastré, 1995; Vidal-Gomel \& Rogalski, 2007), "produit d'un processus historique et collectif» (Samurçay \& Rogalski, 1992, p. 235) transmis par l'expérience faite d'abord avec le chercheur dans les situations dialogiques précédentes et "prêt à servir » dans les situations dialogiques suivantes (Clot, 2008), sans pour autant que cette conceptualisation soit facilement explicitable par les professionnels.

Cette seconde forme d'organisation du dialogue dans laquelle Christophe compare son propre geste au geste de Serge signale selon nous une forme de «migration fonctionnelle » (Vygotski, 2003) en cours, capitale pour l'appropriation de la situation d'autoconfrontation: la comparaison poursuivie par le chercheur dans l'autoconfrontation simple, en l'absence du collègue, devient un moyen de l'activité dialogique de Christophe dans l'autoconfrontation croisée. Ce processus révèle qu'une genèse instrumentale de la comparaison est en cours. C'est un premier palier de 
développement qui marque l'appropriation en cours des contraintes, exigences et ressources propres au dialogue en autoconfrontation dont la comparaison fait partie. Face à la difficulté à dire quelque chose de l'activité d'autrui, le professionnel va puiser dans des ressources qui se sont éventuellement constituées précédemment dans d'autres situations pour les adapter au contexte de la situation en cours. Ici, ces ressources ont pris leur origine, comme on l'a vu plus haut, par l'entremise de l'activité du chercheur lui-même qui insiste de manière répétée et de manière indirecte sur l'importance de la comparaison.

Par conséquent, on peut regarder ce processus comme un passage des ressources sociales extérieures dans l'activité propre du professionnel, qui tente de s'approprier à sa façon ce qu'il a d'abord expérimenté avec le chercheur dans l'autoconfrontation simple. La comparaison qui organise le dialogue et qui s'est d'abord réalisée entre Christophe et le chercheur dans le premier échange, organise et oriente maintenant l'activité même de Christophe dans l'autoconfrontation croisée avec son collègue. D'abord source de l'activité individuelle, la situation sociale d'autoconfrontation simple devient ressource pour le dialogue dans l'autoconfrontation croisée.

La comparaison en cours d'appropriation produit des effets notables dans le dialogue. On peut repérer dans l'extrait ci-dessus comment l'usage de la comparaison par Christophe permet aux deux professionnels de rentrer davantage dans le détail des organisateurs de l'activité pratique (l'habitude, en 35, tirer ou pousser le balai, en 39). En comparant son activité à celle du collègue, il devient nécessaire de s'expliquer avec son activité, en rendant explicite pour soi-même et pour autrui les détails de l'action et ses motifs, ce qui, on l'a vu plus haut, constituait un obstacle pour ces professionnels au début de l'extrait. Lorsque la comparaison s'engage, l'accord tacite de « naturalisation » ne suffit plus et doit être dépassé. La contrainte du cadre dialogique incarné par le chercheur et par la configuration du dispositif pousse alors chacun à en dire davantage que ce qu'il en dit habituellement.

C'est l'expression de la première fonction de la comparaison que nous avons indiquée plus haut : la comparaison permet de faire circuler entre les professionnels les manières de faire mobilisées par chacun. Elle engage chacun dans la recherche de nouveaux arguments qui visent à justifier les différences entre activités en déliant alors les automatismes habituellement incorporés (Tomas, \& Bonnemain, 2018). Ce faisant, ce processus fait apparaître, en négatif, les détails de l'activité dont chacun peut disposer ensuite pour en faire éventuellement quelque chose dans son activité propre.

Mais ce premier développement de l'activité dialogique n'est pas encore abouti. On ne peut pas encore parler de la comparaison comme instrument du dialogue, même si à ce stade elle produit déjà des effets. Pour cela il faut également comprendre comment cette appropriation des ressources sociales qui organisent l'échange peut conduire au développement effectif de l'activité pratique des professionnels concernés. C'est ce qu'on voudrait montrer à partir des extraits suivants.

\subsection{Seconde autoconfrontation croisée : de la comparaison à la « dispute professionnelle »}

60 La seconde autoconfrontation croisée réalisée par Christophe le confronte à l'activité de son collègue David quelques semaines plus tard. Il s'agit cette fois de l'activité de conduite de la benne à ordure ménagère. Christophe et David sont en effet tous deux chauffeurs de 
benne, et sont amenés régulièrement, lorsqu'ils sont affectés sur cette tâche par leur agent de maîtrise, à réaliser une activité de collecte sur le même secteur. La comparaison s'organise ici autour d'un aspect spécifique de cette activité, qui concerne le trajet emprunté pour réaliser la collecte : faut-il par exemple commencer par une rue plutôt qu'une autre pour gagner du temps et de l'efficience ? C'est cet objet de débat qui oriente la conversation entre les deux professionnels dans l'extrait que nous présentons cidessous. L'activité filmée et en cours de diffusion sur l'écran est celle de David. David et Christophe ne s'y prennent pas de la même manière pour organiser leur tournée: Christophe indique aux deux éboueurs à l'arrière de la benne (appelés les « releveurs ») de quel côté de la rue ils doivent ramasser les poubelles, tandis que David les laisse s'organiser entre eux. C'est sur ce point que débute l'extrait.

On verra comment la comparaison maintenant recherchée par Christophe débouche sur une controverse professionnelle, et comment, par suite, on peut repérer des mouvements affectifs dans ce dialogue qui conduiront à un développement effectif de l'activité pratique de David.

Tableau 3 : Extrait d'autoconfrontation croisée entre Christophe et David. Table 3: Extract from crossed self-confrontation between Christophe and David

1 Chercheur : Comment tu fais pour qu'ils fassent ça?

2 Christophe : Ben à force

3 Chercheur : tu leur dis ou alors?

4 Christophe : bah au début ouais mais après

5 Chercheur : Ils font ça tout seuls?

6 David : ben après ils savent au début tu leur expliques

7 Christophe : ben après ils savent je leur dis une fois

8 Chercheur : ouais mais par exemple si t'avais été là avec euh Fabrice et Youcef ce jour-là là toi tu leur a rien dit pour qu'ils fassent ça

9 David : non non non moi suis

10 Christophe : ah ouais mais moi je lui aurais dit à Fabrice je lui aurais dit reste de ce côté-là

11 Chercheur : ah ouais?

12 Christophe : ouais je lui aurais dit... comme moi rue Pierre Legrand avec la nouvelle rue tu peux demander aux releveurs à Serge et tout

13 Chercheur : ouais

14 Christophe :j'en dépose un à la nouvelle rue que lui il va à droite il change tous les sacs tout seul et il y en a un il reste derrière

15 Chercheur : la nouvelle rue c'est là où il $\mathrm{y}$ a les plastiques là ?

16 Christophe : ouais

17 David : ouais

18 Christophe :j'en dépose un j'dépose Serge il fait la rue complète tout seul l'autre releveur il reste derrière le temps que je fais aller-retour comme ça dès que j'arrive à la nouvelle rue Serge est déjà à l'école donc la rue elle est déjà toute entamée parce que pour faire aller-retour t'as besoin de qu'un releveur y'a y'a trois corbeilles c'est tout. Regarde plus loin on va la voir la nouvelle rue.

19 Chercheur : (avance les images) C'est là ? donc là toi tu toi tu envoies euh Serge c'est ça?

20 Christophe : ouais 
21 David : moi ce que j'fais c'est qu'ils enlèvent les sacs y'en a un il enlève les sacs et puis il avance et l'autre il met il met les autres tu vois?

22 Christophe : alors que moi nan... là j'freine

23 David : ouais

24 Christophe : y'en a un il descend

25 Chercheur : ouais

26 Christophe : il s'démerde avec la rue là et l'autre il reste comme ça je fais allerretour là et après je vais rechercher

27 Chercheur : ah d'accord

28 Christophe : tu vois comme ça

29 Chercheur : et l'autre pendant ce temps-là il reprend tous les

30 Christophe : ouais comme ça l'autre il... il fait tout la rue là

31 David : tu vois regardes (pointe les images) comme Fabrice il va prendre les sacs... et il va partir

32 Christophe : toi c'est ce que tu fais toi

33 David : ouais lui il part

34 Chercheur : regardes ouais comment il va faire

35 Christophe : ouais mais après toi tu es embêté tu dois refaire le grand tour pour continuer ta rue

36 David : ben nan parce que même j'fais l'tour j'fais rue comment (.) la rue Ferrer j'la fais en même temps et après je reviens et après j'continue euh

37 Christophe : ouais mais c'est chiant tu reviens sur tes pas

38 David : ouais non ouais

39 Christophe : que là si t'en laisse un tu fais comme ça et tu reviens

40 David : ouais c'est...

41 Chercheur : mais attend on va voir parce que si ça se trouve euh si ça se trouve il $\mathrm{y}$ a des avantages aussi à faire comme ça

42 Christophe : nan parce que tu fais le tour... après tu reviens sur tes pas

43 David : nan c'est parce que tu gagnes au niveau de la rue Ferrer qu'est faite t'es tranquille

44 Christophe : nan, mais moi ça je le fais a- après quand je descends la rue Philadelphie

45 David : ouais ouais donc tu vois

46 Christophe : tu vois quand je fais la rue Philadelphie

47 David : ouais tu- tu vas en face

48 Christophe : je vais tout droit

49 David : ouais

50 Christophe : et après je descends rue d'Aguesseau

51 David : ouais bah c'est ch- ouais j'te dis chacun y fait euh (.) j'te dis on a pas du tout les mêmes euh

52 Christophe : non

53 David : euh façons de travailler et...

54 Chercheur : ouais... mais ça serait quoi l'avant- moi j'ai pas compris l'avantage à faire comme ça là... parce que toi si tu le fais comme ça c'est que...

55 David : ouais c'est parce que c'est moi qui veut le faire comme ça

56 Chercheur : ouais et puis

57 David : tu vois c'est...

58 Chercheur : et puis comme t'as de l'expérience c'est aussi que c'est efficace de 
faire comme ça j'imagine

59 David : ouais ben c'est ben p't'être la sienne elle l'est p't'être aussi parce que j'ai jamais essayé de faire comme lui, mais peut-être que ça s'rait p't'être aussi bien de faire comme il fait Christophe

60 Chercheur : qu'est-ce qu'est-ce que t'en penses toi de sa manière de...

61 David : nan après faut essayer pour voir vraiment euh

62 Chercheur : ouais si ça se trouve c'est intéressant

63 David : ouais ça peut être intéressant hein... mais bon moi après j'ai pris une habitude de travailler comme ça donc euh...j'aime pas trop changer

Dans cet extrait, alors que la conversation s'engage autour des modalités de collecte et de communication avec les éboueurs à l'arrière de la benne (en 3), Christophe rapatrie l'exemple de sa manière de procéder dans «la nouvelle rue » (en 12) pour poursuivre l'argumentation avec son collègue. Après lui avoir explicité cette façon de faire, la controverse professionnelle s'engage entre les deux chauffeurs. Christophe laisse l'un des releveurs seul dans la nouvelle rue, tandis qu'il va effectuer la collecte d'une autre rue. En revenant ensuite dans la nouvelle rue, l'éboueur a eu le temps d'entamer le travail en décrochant les sacs plastiques pleins et en les remplaçant par des neufs. Pour Christophe, c'est un gain de temps important. David, quant à lui, effectue la nouvelle rue en s'engageant sur la voie avec les deux releveurs à l'arrière de la benne. L'un des releveurs ramasse les sacs pleins dans les corbeilles et les jette dans la trémie, tandis que le second releveur le suit pour mettre des sacs plastiques vides et propres.

On peut noter que c'est Christophe lui-même qui pointe à plusieurs reprises les différences entre les deux façons de faire cette même tournée et qui compare l'activité de son collègue à la sienne propre (« moi je lui aurais dit à Fabrice », en 10 ; «alors que moi nan... là j'freine ", en 22 ; «toi c'est ce que tu fais toi », en 32). Il formule également un certain nombre d'objections vis-à-vis de la manière de faire de son collègue (« ouais mais après toi tu es embêté », en 35 ; « ouais mais c'est chiant tu reviens sur tes pas », en 37). Pour nous, cela signale une forme d'appropriation du cadre méthodologique. La comparaison ne constitue plus seulement, comme c'était le cas dans les deux premiers extraits présentés, un moyen de définir un objet de discours. Elle constitue aussi ici un instrument de l'échange, c'est-à-dire un moyen pour Christophe d'affecter son activité et celle d'autrui, un moyen de dépasser le déjà-dit et le déjà-pensé dans la conflictualité réglée avec son collègue.

Les mouvements affectifs provoqués par la comparaison entre activités sont repérables dans le mouvement des gestes, de la voix et des mots (Bonnemain, 2015 ; Bonnemain, \& Clot, 2017; Perrot, 2017; Poussin, 2014). Nous proposons ici une analyse de ces mouvements affectifs dans l'activité dialogique de David pour ensuite montrer les effets de ce dialogue dans son activité pratique ${ }^{2}$. On s'attachera ici à repérer les indices 
émotionnels de réalisation de l'affect au cours d'un moment de l'échange particulièrement significatif selon nous.

L'analyse des indices émotionnels de réalisation de l'affect dans l'activité de David suppose de distinguer trois plans d'analyse distincts, à la suite des travaux réalisés sur cette question dans une perspective développementale (cf. Tableau 4) :

- Les modalités vocales de réalisation de l'affect (Grosjean, 1991): modulations de rythme (silence, "fausses notes ») ; variations intonatives (accentuations, allongements sonores); les rires ;

- Les modalités gestuelles de réalisation de l'affect: gestes co-verbaux qui « véhiculent la composante affectivo-émotionnelle de l'énoncé » (Cosnier, 2003) ; les extra-communicatifs qui peuvent caractériser un inconfort et constituer le signe d'une désorganisation de l'activité ; les variations de postures qui marquent une « rupture » ou un « repli » (Morel, 2006) ;

- Les modalités langagières de réalisation de l'affect: modulations et modalisations qui permettent de rendre compte du rapport entre le locuteur et son propre discours (Vion, 2003) ; les discontinuités discursives qui signalent des « décrochages » dans le fil du discours (Vion, 2003) ; les répétitions.

À partir de cette grille d'analyse, nous analyserons uniquement une courte séquence tirée de l'extrait ci-dessus qui montre l'existence des trois indices émotionnels (vocal, gestuel, langagier) de réalisation de l'affect de manière simultanée dans l'activité de David. Cette simultanéité soutient l'existence d'un affect qui désorganise l'activité dialogique de David et qui le conduit à repenser sa manière de faire la tournée de collecte.

Tableau 4 : convention de codage pour l'analyse des mouvements affectifs dans l'activité de David. Table 4: Coding convention for the analysis of affective movements in David's activity

\begin{tabular}{|c|c|}
\hline Modalité de réalisation de l'affect & Codage pour la transcription \\
\hline \multicolumn{2}{|l|}{ VOIX } \\
\hline Accents d'insistance / fausse note & en majuscule : « JE suis " \\
\hline \multirow[t]{2}{*}{ Silence } & $($ () \\
\hline & et selon la durée : $(.).(\ldots)$ \\
\hline \multirow[t]{2}{*}{ Pause sonore } & allongement du mot : « eu:h» \\
\hline & et selon la durée : :: : $:$ : \\
\hline Rires & (rires) \\
\hline Répétitions & Souligne : je suis je suis \\
\hline \multicolumn{2}{|l|}{ GESTUALTIE } \\
\hline Modifications posturales & Surligne en bleu : “je suis $\#$ \\
\hline Gestes co-verbaux & Surligné en vert : je suis nx \\
\hline Gestes extracommunicatifs d'inconfort & Surligné en rouge : Ecius: \\
\hline \multicolumn{2}{|l|}{ LANGAGE } \\
\hline Reprise & En gras \\
\hline Modalisation/modulation/discontinuité & En italique \\
\hline
\end{tabular}


67 La séquence analysée débute en 42 , alors que Christophe poursuit la comparaison entre son activité et celle de David. Elle se termine en 55. Elle regroupe l'ensemble des indices émotionnels de réalisation de l'affect et elle est à ce titre significative des mouvements affectifs qui se réalisent dans l'activité dialogique de David, au cours de la controverse professionnelle entre les deux éboueurs.

Tableau 5 : Les indices émotionnels de réalisation de l'affect dans l'activité de David. Table 5: Emotional indices for realisation of the affect in David's activity

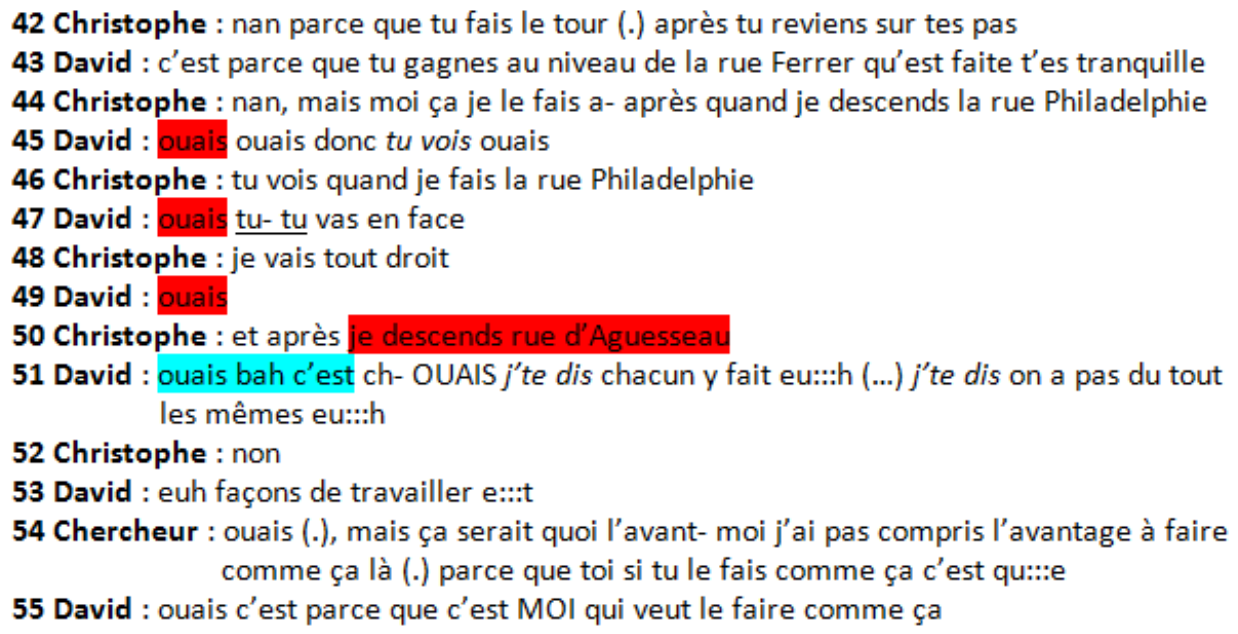

Cette courte séquence réunit l'ensemble des indices émotionnels de réalisation d'un affect. Alors que Christophe est engagé dans la comparaison entre les deux activités, David est poussé à justifier ses modalités d'action auprès de lui et auprès du chercheur. Christophe formule explicitement des objections vis-à-vis de la manière de faire de David. En 43, David rentre dans l'argumentation avec son collègue (« c'est parce que tu gagnes au niveau de la rue Ferrer »). Mais Christophe, en 44, avance un nouvel argument en comparant à nouveau les deux activités (" nan, mais moi ça je le fais après »). C'est cette insistance dans l'argumentation à laquelle réagit David, en 45, par la réalisation d'un geste d'inconfort en penchant la tête sur le côté droit tout en levant les sourcils. On fait l'hypothèse ici qu'il a le sentiment d'être poussé dans ses retranchements par son collègue, ce qui semble être une situation perturbante pour lui. C'est pourquoi en 47 il réitère ce geste d'inconfort de la tête. Christophe poursuit ensuite en 48 la description de sa manière de faire qui sera ratifiée par David en 49. Cet énoncé est à nouveau associé au même geste de la tête. En trois tours de parole, ces trois gestes que l'on qualifie ici de gestes d'inconfort semblent montrer qu'une perturbation est en cours dans l'activité de David.

Mais c'est en 51 que cet affect semble le plus intense. Face à l'argumentation de son collègue, David ne semble plus trouver les mots qui lui permettraient de poursuivre l'échange. Le segment «ouais bah c'est ch- OUAIS j'te dis chacun y fait eu:::h (...) » est significatif d'une perturbation de la voix associée à l'affect en cours de réalisation. C'est ce que nous avons qualifié plus haut de "fausse note », qui marque le tumulte émotionnel qui désorganise l'activité de David sur le plan vocal. À la fin de ce segment, David réalise également une pause de trois secondes avant de poursuivre l'énoncé ( « (...) j’te dis on a pas du tout les mêmes eu:::h »), qui caractérise une perturbation langagière provoquée par un dialogue intérieur très intense qui l'empêche de poursuivre son discours extériorisé de 
manière simultanée. Les allongements sonores « eu:::h » semblent également confirmer cette hypothèse d'un dialogue intérieur simultané.

Mais ces indices émotionnels de désorganisation vocale sont également associés à une modification simultanée de sa posture (surligné en bleu) vers une posture "bras croisés » que l'on peut qualifier, avec Morel (2006), de «posture de repli » caractéristique d'un éprouvé affectif.

71 Notons également la réaction du chercheur, en 54, qui tente de soutenir l'activité dialogique de David visiblement perturbé par la situation («j'ai pas compris l'avantage à faire comme ça là »). Par cette relance, le chercheur tente de soutenir la manière de faire de David face à celle de Christophe qui semble avoir pris l'ascendant dans l'argumentation. Il cherche à le pousser à rendre plus explicites les «avantages » de sa manière de faire. Ce faisant, il cherche également à soutenir l'affectivité palpable dans la situation afin de réhabiliter la manière de faire de David qui, a priori, n'est pas moins efficace que celle de Christophe.

72 L'ensemble de ces éléments soutient selon nous l'existence d'un affect dans l'activité de David.

73 David est affecté par l'alternative que son collègue décrit qui vient bousculer sa propre expérience de la collecte. L'expérience qu'il a est prise en défaut par l'expérience qu'il fait dans le dialogue avec son collègue Christophe. L'énoncé en 51 , «on a pas du tout les mêmes façons de travailler » montre comment David tente de rapatrier l'expérience qu'il fait là à du " déjà vécu ", ou à rendre le problème de l'efficacité entre les deux manières de faire soluble dans une "naturalisation » des différences entre activités. Le fait que "chacun fait comme il veut» est un discours convenu que l'on retrouve très fréquemment dans ce milieu professionnel, et dans d'autres, un « déjà-dit » qui souligne la passivité associée à l'affect à ce stade de la conversation.

74 Mais la reprise de l'échange par le chercheur, qui tente de soutenir l'expérience de David (« comme tu as de l'expérience c'est aussi que c'est efficace de faire comme ça », en 58), amène David à envisager la possibilité de s'essayer à la manière de faire de Christophe. Repris dans l'échange par le chercheur, l'affect est sans doute rendu plus supportable pour David. C'est pourquoi il peut envisager ensuite d'expérimenter la manière de faire de son collègue (en 61).

75 Mais cette forme d'activité retrouvée dans l'échange n'est que transitoire, la fin de l'extrait montre que l'activité de David est prise dans une oscillation entre passivité et activité. L'énoncé en 63 caractérise pour nous ce mouvement de balancier affectif (« ouais ça peut être intéressant hein... mais bon moi après j'ai pris une habitude de travailler comme ça donc euh... j'aime pas trop changer »). Nous verrons ensuite que ces mouvements affectifs ne présagent pas du devenir de ce dialogue dans l'activité pratique. Malgré la passivité qui affleure, mais qui, comme on l'a vu, n'est pas définitive, les professionnels peuvent très bien réussir à en faire quelque chose dans leur activité pratique.

76 Quoi qu'il en soit, c'est dans la traversée des différentes situations que nous avons présentées que Christophe a pu affuter sa capacité à repérer les différences entre les activités et à en faire usage dans le dialogue avec ses collègues. Mais ce repérage ne peut être réduit à une forme d'inventaire, ou à une catégorisation cognitive. Elle prend inévitablement sa source dans le conflit affectif de l'activité (Bonnemain, 2015; Clot, 2017). Dans l'extrait présenté ci-dessus, David est également amené à repérer ces 
différences, poussé par son collègue qui soutient le désaccord avec sa manière de faire. Or, comme l'a bien vu Moscovici (2005):

«Chacun éprouve une tension, a le sentiment d'un trouble ou d'un déplaisir, lorsqu'il se trouve exposé à une opinion ou à une évaluation qui diffère de la sienne

(...) La discorde et tout conflit en général sont difficiles à surmonter par les individus » (p. 145).

C'est pourquoi, en faisant de la comparaison un instrument de l'échange avec ses collègues, Christophe expose son collègue et s'expose lui aussi à la conflictualité sur les critères de la qualité du travail. C'est là un engagement sensible dans le dialogue qui n'a plus grand-chose à voir avec la recherche d'un soutien apporté au recueil des données par le chercheur, comme c'était le cas dans l'autoconfrontation simple. L'analyse de son activité, d'abord adressée au chercheur, s'adresse maintenant à son collègue de travail au nom de ce qui peut être jugé efficace ou non, c'est à dire au nom de la qualité du travail.

Résumons-nous : le processus d'appropriation de la situation d'autoconfrontation est un processus d'appropriation des artefacts dialogiques au service du développement de l'activité de pensée. Parmi ces artefacts, la comparaison nécessite d'être appropriée par les professionnels eux-mêmes pour que le dialogue puisse éventuellement se muer en controverse professionnelle, source de mouvements affectifs qui peuvent avoir un destin dans l'activité pratique. Ainsi, on a vu jusqu'à présent à partir de l'exemple de Christophe que la comparaison naît d'abord comme un objet d'échange avec le chercheur dans l'autoconfrontation simple, elle devient ensuite un moyen pour Christophe de conduire le dialogue dans l'autoconfrontation croisée avec Serge, et elle peut éventuellement s'y muer en controverse professionnelle comme c'est le cas dans le dernier extrait que nous avons présenté avec Didier, à la recherche d'une efficacité renouvelée. C'est ce processus développemental qui décrit selon nous le processus d'appropriation de la comparaison comme instrument du dialogue dans l'autoconfrontation.

\subsection{De la « dispute professionnelle » au développement de l'activité pratique}

Mais il faut également regarder ce dernier extrait dans ce qu'il a de moteur pour le développement de l'activité pratique. Ici, le dialogue comme collision a des effets bien audelà de la situation d'autoconfrontation, dans l'activité pratique elle-même. À l'issue de cette genèse instrumentale de la comparaison dans l'activité de Christophe, le dialogue affecté produit des effets. On peut les repérer dans les traces recueillies ultérieurement au cours de cette intervention. C'est le cas ici dans un échange entre Christophe et David ayant eu lieu un mois après l'autoconfrontation croisée entre eux, lors de la présentation de cette séquence au collectif élargi, en l'absence de la hiérarchie. Nous rapportons ici un court extrait de cette discussion en présence de l'ensemble des éboueurs de l'équipe. Le film est en cours de visionnage. L'échange débute à la fin de la séquence. Un échange avec les collègues s'en suivra ensuite, mais nous rapportons ici uniquement la discussion entre Christophe et David dans un souci de synthèse.

Tableau 6 : Extrait de dialogue entre Christophe et David après l'autoconfrontation croisée. Table 6: Extract from dialogue between Christophe and David following crossed self-confrontation

David : (aux collègues) c'est vrai que j'ai essayé comme Christophe, c'est vrai que c'est mieux

Christophe : t'as vu c'est mieux hein?

David : ouais tu perds moins de temps... Maintenant je fais comme ça ouais 
Christophe : et tu laisses le releveur avec seulement deux sacs

David : pour le Grec ouais ça suffit

Chercheur : ah oui tu fais comme ça maintenant?

David : oui parce que quand tu arrives après le releveur il est déjà à l'école donc ça va plus vite

79 Ce court extrait nous permet d'illustrer le devenir potentiel des dialogues réalisés, au moyen de la comparaison, dans l'activité pratique des professionnels qui s'engagent dans l'analyse de leur travail en autoconfrontation. C'est à la condition d'une appropriation de la situation dialogique de l'autoconfrontation que les professionnels peuvent éventuellement retoucher leurs manières de faire. Mais on l'a vu, cette appropriation, dans le cas de la comparaison, n'est pas mécanique, elle passe par une répétition du rapport à autrui et à l'objet de son travail qui produit de l'affect. Or, ces affects constituent une véritable «force de travail» (Green, 1973). Ils sont potentiellement moteurs pour la modification de l'action habituelle.

80 À partir du dialogue en autoconfrontation croisée réalisé avec Christophe, David a fait de l'expérience vécue à cette occasion un moyen de vivre une nouvelle expérience, cette fois-ci dans le cadre de son activité pratique, ce qui définit pour nous une forme de développement. Le dialogue affecté a régénéré le réel de son activité pratique, en faisant apparaître à David une nouvelle variante exploitable dans l'organisation de la tournée. Or, ces variantes, ces autres possibilités non réalisées, sont toujours plus ou moins fossilisées, plus ou moins disponibles. Les autres activités possibles, pour se réaliser, supposent un investissement de la part du sujet et un développement de sa mobilité interfonctionnelle, c'est-à-dire sa capacité à mettre une fonction au service d'une autre, ses émotions par exemple au service de sa pensée et inversement. Dans l'autoconfrontation, ces migrations constituent un instrument décisif pour donner un destin dynamique et actif à l'affect (Bonnemain, \& Clot, 2017).

81 L'appropriation de la situation par Christophe est reliée à ce développement dans l'activité de David. C'est parce que Christophe est en capacité de «supporter» la conflictualité sociale sur les critères de qualité du travail - de poursuivre la comparaison dans le dialogue, soutenu par le chercheur - que du nouveau peut se produire dans le discours. En faisant l'expérience répétée de la délibération professionnelle autour des critères de qualité du travail, il est un peu mieux équipé pour penser à nouveaux frais sa propre activité ou pour discuter celle de ses collègues. C'est d'ailleurs ce qui explique la place prise ensuite par Christophe dans cette intervention. À l'issue de la phase de travail en autoconfrontations, il a été élu par ses collègues de travail en tant qu'opérateur " référent-métier », chargé de représenter le travail ordinaire de manière régulière - une fois par mois - auprès de la hiérarchie, pour traiter les problèmes de travail qui nécessiteraient d'être résolus selon le collectif. Son activité a donc fait l'objet d'un développement dans l'organisation que nous ne pouvons reprendre ici plus en détail, car il nécessiterait l'analyse d'un matériau plus élargi, mais qui est en lien avec son appropriation des artefacts dialogiques de l'autoconfrontation.

82 C'est grâce et seulement au travers de cette appropriation que le dialogue peut dépasser le déjà-dit, et, par suite, engager des modifications concrètes des manières de faire. Mais cette appropriation, on l'a vu, est prise et déterminée par le rapport à autrui dans le cours des autoconfrontations, qu'il s'agisse du chercheur ou du collègue. Ce sont les contraintes imposées par le cadre méthodologique et incarnées en premier lieu par le chercheur qui 
permettent à cette appropriation de devenir effective. À l'issue de cette appropriation, le professionnel peut se mettre à «employer à son propre égard les formes mêmes de conduite que les autres ont employées en premier lieu envers lui» (Vygotski, 1978, p.141). En l'absence de ces contraintes, les professionnels tendent à chercher le consensus comme l'a également à nouveau bien vu Moscovici (2005) dans un autre champ : «Le consensus paraît nécessaire pour vivre en harmonie et se sentir membre d'un groupe, toute dissension étant menaçante pour soi et pour les autres » (p. 145). Ainsi, les contraintes instrumentales du dialogue, comme la nécessité d'engager des comparaisons entre ma manière de faire et celle d'autrui, si elles peuvent constituer d'abord une source de décontenancement, sont en même temps toujours une source potentielle de dépassement des discours habituels, à condition que le chercheur puisse " tenir » sur le cadre méthodologique. On peut parler à ce titre de l'autoconfrontation comme une véritable « situation sociale de développement » (Vygotsky, 1998). C'est sur ce point que nous conclurons.

\section{Discussion : la comparaison entre activités comme condition du développement dans l'intervention} avec l'autoconfrontation et avec l'intervention en général. Elle constitue dans ce cadre la condition d'un développement possible. Sans elle, le dialogue perd en profondeur et en authenticité (Quillerou \& Clot, 2013). En effet, on l'a vu au travers de l'analyse des dialogues ci-dessus, la comparaison remplit deux fonctions essentielles pour le développement: une première fonction de circulation des manières de faire entre professionnels d'un milieu, et une seconde fonction, affective, qui est une source potentielle de disputes professionnelles sur des activités comparables. La comparaison constitue alors l'instrument d'un dialogue affecté entre les professionnels à la recherche de nouvelles manières d'arbitrer les conflits de l'activité dans les situations réelles de travail, ou dans l'organisation avec la hiérarchie.

deux fonctions ne peuvent produire leurs effets jusqu'au développement effectif qu'en lien avec le cadre de travail qui est d'abord tenu et dirigé par le chercheur. C'est lui qui doit indirectement, par ses relances et sa manière de faire, les susciter, les proposer et les "tenir» afin que les professionnels puissent faire une expérience de dialogue singulière qui contraint l'objet de l'échange autour des différences entre activités. Le protocole de travail proposé porte également cette exigence en lui-même, en organisant "physiquement» la comparaison.

C'est grâce à cette possibilité de tenir le cadre du dialogue que l'appropriation de la comparaison devient possible et qu'elle peut ensuite devenir une source des controverses professionnelles dans lesquelles, on l'a vu, l'affectivité joue un rôle moteur pour le développement de la pensée et de l'activité pratique.

La comparaison comme artefact ne garantit pas sa genèse instrumentale dans l'activité dialogique des professionnels. Ils peuvent même tenter de tout faire pour éviter de comparer leurs activités, en rapatriant le déjà-dit et le déjà-vécu comme seule forme d'explication possible des différences entre activités, comme on l'a vu plus haut. C'est pourquoi, face à ces formes de passivité si fréquentes en milieu de travail (Bonnefond, 2016), il s'agit pour l'intervenant de «tenir bon » sur le cadre (Duboscq \& Clot, 2010) et 
sur ses moyens artefactuels, pour que les professionnels puissent s'en approprier quelque chose dans la poursuite des dialogues. Autrement dit, l'enrichissement des conflits de critères passe par la " genèse instrumentale » de l'intervention comme méthode indirecte (Litim, 2012), au sein de laquelle la question de pouvoir comparer son activité à celle d'autrui dans l'autoconfrontation occupe une place importante, mais dont le résultat n'est jamais garanti à l'avance.

Lorsqu'elle peut être appropriée par les professionnels engagés dans l'intervention, la comparaison constitue la source de mouvements affectifs qui peuvent conduire à la réorganisation de l'activité pratique habituelle.

Lors des phases ultérieures de l'intervention, ces expériences dialogiques réalisées et leurs effets constituent une ressource pour engager les échanges avec la hiérarchie sur la réorganisation du service. Ces dialogues montrent l'initiative possible des opérateurs de terrain artificiellement révélée grâce au cadre spécifique d'autoconfrontation. L'organisation, paradoxalement, ne tire pas suffisamment profit de cette initiative possible pour la performance et pour la santé, alors qu'elle permet de faire le tour des questions complexes qui se posent dans les situations réelles de travail. C'est là le point de départ d'un dialogue élargi dans l'organisation avec les décideurs, les professionnels concernés et les organisations syndicales: à quelles conditions peut-on instituer durablement ces dialogues dans l'organisation? Dans l'intervention que nous avons présentée, ces échanges ont conduit à une réorganisation complète et formalisée du dialogue au sein du service de propreté. Les échanges engagés ont permis aux éboueurs de faire davantage autorité auprès des décideurs sur leur travail quotidien et sur les problèmes qui s'y posent. Il ne s'agit pas de considérer que les éboueurs réussissent toujours à construire les problèmes de la bonne manière, ni de considérer que l'échange entre eux produirait toujours les meilleures solutions, il s'agit plutôt de faire en sorte que les diagnostics qu'ils peuvent faire collectivement sur les obstacles à la qualité du travail puissent trouver de nouveaux lieux de délibération avec la hiérarchie dans l'organisation, afin que de nouvelles solutions puissent être imaginées. C'est dans cette "coopération conflictuelle " (Trentin, 2012) à construire dans l'intervention que du nouveau peut se produire, et c'est aussi dans cette coopération nouvelle que le dialogue peut s'instituer dans l'organisation de manière durable autour de la qualité du travail. Mais cette coopération n'est possible que lorsque l'expérience d'un dialogue inédit peut s'initier d'abord entre les professionnels, et dans laquelle la comparaison entre activités revêt une importance majeure.

\section{Conclusion : l'autoconfrontation comme situation sociale de développement}

On peut maintenant revenir sur l'objet qui nous a préoccupé au départ. L'apprentissage, compris ici comme une appropriation/transformation des règles et des artefacts de la situation, est relié au développement dans le temps. Pour qu'un développement puisse se produire, il faut que les professionnels, chacun à leur manière, s'approprient les contraintes et ressources du dialogue proposé pour en faire quelque chose pour euxmêmes et entre eux. Il y a un devenir de l'apprentissage dans le développement. Cette appropriation, on l'a vu, s'organise dans un mouvement du dehors vers le dedans, d'une relation sociale avec le chercheur dans l'autoconfrontation simple, qui fait de la comparaison une règle de fonctionnement spécifique en la suscitant, jusqu'à une 
technique propre au sujet, à la recherche de quelque chose de neuf dans l'échange avec son collègue. Dans notre exemple, c'est ce genre d'appropriation, lorsqu'elle est possible, qui permet aux dialogues d'aller au-delà des discours habituels et qui engage des transformations de l'activité pratique.

90 À travers ce processus, on peut du coup regarder l'autoconfrontation comme une situation sociale de développement dans l'intervention. Elle «représente le point de départ de tous les changements dynamiques qui se produisent dans le développement pendant une période donnée » (Vygotsky, 1998), même si ce n'est pas la seule situation sociale de l'intervention (comités de pilotage avec la hiérarchie, comités de suivis avec les organisations syndicales).

91 Dans ce genre de situation sociale spécifique, « les caractéristiques d'une personnalité en mouvement rencontrent les attentes et les possibilités ouvertes par l'interaction avec l'environnement, dont elle perçoit les attentes et les ressources, en est affectée, et fait quelque chose de, sur et avec cet environnement » (Mayen, \& Olry, 2012).

À la suite de ce que nous avons présenté, on pourrait dire que le cadre méthodologique de l'autoconfrontation a permis à Christophe de faire quelque chose des contraintes dialogiques que ce dernier lui impose. Cette situation "donne une certaine place, fonction et signification aux artefacts qui la composent et aux artefacts en action qu'exigent les actions adéquates à la situation : objets, manières de faire, jeux de langage, modes de raisonnement, concepts, etc.» (Mayen, 2012, p. 304). Elle est toujours potentiellement développementale. Mais rien ne garantit à l'avance la réalisation de développements effectifs. Il y faut également « des formes de médiation humaine et une configuration personnelle et sociale en mouvement qui pousse les personnes à chercher à s'approprier ces situations» (Ibid.). Dans l'autoconfrontation, la comparaison comme contrainte - comme artefact - peut devenir ensuite un instrument de l'activité du professionnel lorsqu'il observe l'activité de ses collègues pour en dire quelque chose. Mais cette genèse instrumentale, pour se réaliser, doit pouvoir être médiée par l'activité du chercheur qui peut la susciter, la renforcer ou la favoriser. C'est seulement à cette condition, c'est-à-dire lorsque la comparaison constitue l'objet du rapport social entre deux personnes, qu'elle devient éventuellement ensuite une ressource propre : d'abord sur le plan social, puis sur le plan psychologique. Mais l'inverse est aussi vrai: la comparaison, en tant qu'instrument psychologique de conduite du dialogue, est retouchée par le sujet pour résoudre un problème ou poursuivre un questionnement qui le mobilise. Elle peut alors faire l'objet d'une stylisation qui en retour, affecte potentiellement les collègues, et plus largement le milieu professionnel.

Nous avons présenté dans cet article une forme possible du processus d'appropriation de la comparaison chez un professionnel singulier, mais il peut exister d'autres formes d'appropriation des artefacts dialogiques reliés à la situation d'autoconfrontation. La situation sociale de développement étant par définition une rencontre entre "les conditions externes de la situation et la logique interne de la dynamique de la vie professionnelle d'une personne (ses motifs, ses capacités) ( (Mayen, \& Olry, 2012), cela dépend donc également de l'histoire des sujets qui s'engagent toujours pour différentes raisons dans ce genre de dispositifs. C'est pourquoi le développement de l'activité pratique est toujours seulement potentiel. Il est imprédictible, mais pas inexplicable. Il dépend des artefacts contenus par le cadre méthodologique que les professionnels peuvent s'approprier en coopération avec le chercheur. Il dépend tout autant de la demande et des attentes des professionnels qui sont parfois à la recherche d'autres 
possibilités pour agir. Dans ce contexte, on peut regarder l'autoconfrontation comme une situation sociale qui peut équiper la rencontre entre ces deux conditions du développement.

\section{BIBLIOGRAPHIE}

Bakhtine, M. (1970). L'œuvre de François Rabelais et la culture populaire au moyen âge et sous la renaissance. Paris : Gallimard.

Bakhtine, M. (1984). Esthétique de la création verbale. Paris : Gallimard.

Bakhtine, M. (2003). Pour une philosophie de l'acte. Lausanne : Editions l'Age d'Homme.

Barcellini, F. (2017). Intervention Ergonomique Capacitante : bilan des connaissances actuelles et perspectives de développement. Activités, 14(2). https://journals.openedition.org/activites/3041

Bender, C. (1998). Bakhtinian perspectives on "everyday life" sociology. In, M. M. Bell \& M. Gardiner (Eds). Bakhtine and the Human Sciences (p. 181-195). London: Sage Publications.

Bonnefond, J.-Y. (2016). L'intervention dans l'organisation en clinique de l'activité. Le dispositif "DQT" à l'usine Renault de Flins. Thèse pour le Doctorat en psychologie. Paris : Cnam.

Bonnefond, J.-Y. (2017). Intervention et développement organisationnel en clinique de l'activité. Activités, 14(2). https://journals.openedition.org/activites/3014

Bonnefond, J.-Y., \& Clot, Y. (2016). Les affects et leur destin dans l'intervention. Un exemple dans l'industrie automobile. Activités, 13(2). https://journals.openedition.org/activites/2895

Bonnefond, J.Y., Bonnemain, A., Fontes, F., \& Clot, Y. (2016). To institute conflictive cooperation on the quality of work. Educação, 39, 42-53.

Bonnemain, A. (2015). Les paradoxes de l'intensité affective dans l'autoconfrontation. L'exemple de l'activité dialogique des chefs d'équipe de la propreté de Paris. Thèse pour le doctorat en psychologie. Paris : Cnam.

Bonnemain, A., Bonnefond, J.Y., Fontes, F.F., \& Clot, Y. (sous presse). Vygotski and work: an activity clinic to change the organization. In P.F. Bendassolli (Ed.), Culture, work and psychology: invitation to dialogue (p. 59-80). Information Age Publishing.

Bonnemain, A. \& Clot, Y. (2017). Clinique de l'activité : les affects dans l'autoconfrontation. In M. Santiago-Delefosse \& M. del Rio Carral (Eds.), Les méthodes qualitatives en psychologie (p. 131-151). Paris : Dunod.

Canguilhem, G. (2002). Ecrits sur la médecine. Paris : Seuil.

Carta, G., \& Falzon, P. (2017). Co-construire l'autopoïèse organisationnelle : le Laboratoire Développemental comme modèle et comme moyen de l'intervention capacitante. Activités, 14(2). https://journals.openedition.org/activites/3022

Clot, Y. (2008). Travail et pouvoir d'agir. Paris : PUF.

Clot, Y. (2017). L'affectivité en activité. In J.-M. Barbier \& M. Durand (Eds.), Encyclopédie d'analyse des activités (p. 877-910). Paris : PUF. 
Clot, Y., \& Faïta, D. (2000). Genres et styles en analyses du travail : concepts et méthodes. Travailler, 4, 7-43.

Clot, Y., Faïta, D., Fernandez, G., \& Scheller, L. (2001). Les entretiens en auto-confrontation croisée : une méthode en clinique de l'activité. Éducation permanente, 146, 17-27.

Cosnier, J. (2003). Les deux voies de communication de l'émotion (en situation d'interaction de face à face). In J-M. Colletta, \& A. Tcherkassov (Eds.), Les émotions. Cognition, langage et développement (p. 59-68). Sprimont : Mardaga.

Duboscq, J., \& Clot, Y. (2010). L'autoconfrontation croisée comme instrument d'action au travers du dialogue : objets, adresses et gestes renouvelés. Revue d'anthropologie des connaissances, 2 , 255-286.

Faïta, D. (1999). Analyse des situations de travail : de la parole au dialogue. In J. Richard-Zappella (Ed.), Espaces de travail, espaces de parole (p. 127-136). Collection Dyalang.

Faïta, D. (2011). Théorie de l'activité langagière. In B. Maggi (Ed.), Interpréter l'agir : un défi théorique (p. 41-68). Paris : PUF.

Falzon, P. (2013). Ergonomie constructive. Paris : PUF.

François, F. (1998). Le discours et ses entours. Paris : L'Harmattan.

Green, A. (1973). Le discours vivant. Paris : PUF.

Grosjean, M. (1991). Les musiques de l'interaction. Contribution à une recherche sur les fonctions de la voix dans l'interaction. Thèse de doctorat en psychologie. Université de Lyon.

Hochschild, A. R. (2003). Travail émotionnel, règles de sentiments et structure sociale. Travailler, 9 (1), 19-49.

Kostulski, K. (2004). Développement de la pensée et du rapport à l'autre dans une interlocution : "est-ce que c'est un endroit pour poser un paquet de contre-rail ?". Cahiers de linguistique française , 26, 113-131.

Kostulski, K., \& Clot, Y. (2007). Interaction et migration fonctionnelle : un développement en autoconfrontation croisée. In Y. Clot, \& K. Kostulski (Eds.), Dialogue, activité, développement. Psychologie de l'interaction, 23-24, 73-109.

Leplat, J. (2014). Aperçu sur les critères à la lumière de l'histoire. In D. Lhuilier (Ed.), Qualité du travail, qualité au travail (p. 17-26). Toulouse : Octarès.

Litim, M. (2012). Les méthodes indirectes à l'épreuve de la pratique : questions d'intervention. In Y. Clot (Ed.), Vygotski maintenant (p 165-175). Paris : La Dispute.

Livet, P. (2007). Emotions et révision : la dynamique des débats. In C Blatrix et al. (Eds.), Le débat public : une expérience française de démocratie participative (p. 339-352). Paris : La Découverte.

Massot, C., \& Simonet, P. (2017). Intervenir dans l'entreprise pour soutenir la discussion sur le travail comme moyen de transformation. Perspectives interdisciplinaires sur le travail et la santé, 19 (3).

Mayen, P. (2012). L'appropriation des situations. In Y. Clot (Ed.), Vygotski maintenant (p. 289-305). Paris : La Dispute.

Mayen, P., \& Olry, P. (2012). Expérience du travail et développement pour de jeunes adultes en formation professionnelle. Recherche et formation, 70, 91-106.

Morel, M.-A. (2006). Un thème, deux thèmes, un préambule ? Intonation, geste et morphosyntaxe dans le dialogue oral en français. Linx, 55, 133-152. 
Moscovici, S. (Ed). (2005). Psychologie sociale des relations à autrui. Paris : Armand Colin.

Pastré, P. (2012). La didactique professionnelle. Approche anthropologique du développement chez les adultes. Paris : PUF.

Perrot, E. (2017). L'activité transférentielle, une ressource au service du développement du métier. Le cas des agents d'escale et de service commercial en gare. Thèse pour le doctorat de psychologie. Paris : Cnam.

Peytard, J. (1990). Évaluation sociale dans les thèses de Mikhaill Bakhtine et représentations de la langue. Langue Française, 85(85), 6-21.

Poussin, N. (2014). Développement des sentiments au travail : dialogues sur l'efficacité et l'utilité chez les médecins du travail. Thèse pour le doctorat de psychologie. Paris : Cnam.

Quillerou, E., \& Clot, Y. (2013). Trois conditions pour une clinique de l'activité en psychologie du travail : le cas d'une intervention dans une entreprise de logistique automobile. Activités, 10(2), 229-248. https://journals.openedition.org/activites/842

Ribert-Van de Weerdt, C. (2011). Les contraintes de travail et les stratégies de régulation émotionnelle en centre de relation clientèle. Le Travail Humain, 74(4), 321-339.

Rocha, R., Mollo, V., \& Daniellou, F. (2017). Le débat sur le travail fondé sur la subsidiarité : un outil pour développer un environnement capacitant. Activités, 14(2). https:// journals.openedition.org/activites/2999

Samurçay, R., \& Pastré, P. (1995). La conceptualisation des situations de travail dans la formation des compétences au travail. Éducation permanente, 123, 34-42.

Samurçay, R., \& Rogalski, J. (1992). Formation aux activités de gestion d'environnements dynamiques : concepts et méthodes. Éducation permanente, 111, 227-242.

Thébault, J. (2013). La transmission professionnelle : processus d'élaboration d'interactions formatives en situation de travail. Une recherche auprès des personnels soignants dans un Centre Hospitalier Universitaire. Thèse pour le doctorat d'Ergonomie. Paris : Cnam.

Tomas, J.-L., \& Bonnemain, A. (2018). Les gestes en autoconfrontation croisée : enjeux et pratiques. Pratiques psychologiques.

Trentin, B. (2012). La cité du travail. Le fordisme et la gauche. Mayenne : Institut d'Études Avancées de Nantes / Fayard.

Veresov, N. (2014). Emotions, perezhivanie et développement culturel : le projet inachevé de L. Vygotski. In C. Moro \& N. Muller Mirza (Eds.), Sémiotique, culture et développement psychologique (p. 209-235). Villeneuve d'Ascq : Presses Universitaires du Septentrion.

Vidal-Gomel, C., \& Rogalski, J. (2007). La conceptualisation et la place des concepts pragmatiques dans l'activité professionnelle et le développement des compétences. Activités, 4(1). https:// journals.openedition.org/activites/1401

Vion, R. (2003). Expression et gestion des émotions dans les interactions verbales. In J.M. Colletta, \& A. Tcherkassof (Eds.), Les émotions. Cognition, langage et développement (p. 153-158). Sprimont : Mardaga.

Vygotsky L. (1978). Mind in society: the development of higher psychological processes. Cambridge, Harvard University Press.

Vygotsky, L. (1998). The collected Works of L. S. Vygotsky, vol. 5: Child Psychology (1929). New York : Plenum Press. 
Vygotski, L. (2003). Conscience, inconscient, émotions. (F. Sève \& G. Fernandez, Trad). Paris : La Dispute.

Vygotski, L. (2014). Histoire du développement des fonctions psychiques supérieures. Paris : La Dispute. Yvon, F., \& Clot, Y. (2003). Apprentissage et développement dans le travail enseignant. Pratiques psychologiques, 1, 19-35.

\section{NOTES}

1. Le lecteur pourra se reporter à cet article pour une description précise des différentes phases de la méthode de l'autoconfrontation en clinique de l'activité, ce que nous ne pouvons prendre le temps de faire ici. On y trouvera écrites les conditions dans lesquelles ce genre de dialogue peut être installé en milieu de travail.

2. Nous ne présenterons pas les mouvements affectifs associés à l'activité de Christophe dans le cadre de cet article et dans un souci de synthèse, même si ces mouvements existent également.

\section{RÉSUMÉS}

Cet article traite de l'appropriation par les professionnels de l'une des modalités du dialogue en autoconfrontation dans une perspective en clinique de l'activité. Cette modalité concerne la manière dont les professionnels peuvent faire de la comparaison entre leurs activités professionnelles un moyen de l'échange entre eux. Cela n'est pas un processus spontané, mais un résultat du développement de leur activité dialogique dans différents contextes que nous décrirons, et dans lesquels l'affectivité joue un rôle décisif. Dans le cadre contraint de l'autoconfrontation, s'opèrent parfois des formes de genèses instrumentales de la comparaison qui devient alors un organisateur de l'activité dialogique. L'appropriation de ce moyen dialogique par les professionnels équipe les échanges et permet de dépasser les discours habituels socialement partagés sur le travail et les problèmes concrets qui s'y posent. La comparaison constitue alors un moyen du développement de l'activité au travers du dialogue, ce qui suppose une activité d'intervention qui tienne compte de ces mouvements affectifs. À partir de l'exemple clinique d'une intervention réalisée auprès des éboueurs de la Ville de Lille, nous montrerons que lorsque ce genre de comparaison s'installe dans l'autoconfrontation, elle peut engager, en lien avec les mouvements affectifs qui s'y réalisent, des développements de l'activité professionnelle qui permettent du coup de regarder la méthode comme une «situation sociale de développement » (Vygotsky, 1998).

The purpose of this article is to analyze the process of appropriating dialogue in a clinic activity perspective. This process relates to how professionals can make a comparison between their activities a means of dialogue, which is not a spontaneous process, but a result of dialogical development in the different contexts that we will describe. Affectivity plays a central role in this process. In the constrained framework of crossed self-confrontation, there may be some forms of instrumental genesis of comparison which become organizers of dialogic activity. Appropriation of this dialogical means by professionnals equips dialogues and makes it possible to go beyond the standard socially shared discourses on work and its associated problems. Comparison then 
becomes a way to affect one's own activity or that of others in dialogue. This supposes an intervention activity that takes these affective movements into account. We will use a clinical example to show that when this kind of comparison takes place within crossed selfconfrontation, it can engage developments of professional activity, which thus make itpossible to view the self-confrontation method as a "social situation of development" (Vygotsky, 1998).

INDEX

Mots-clés : affect, comparaison, autoconfrontation, dialogue

Keywords : affect, comparison, crossed self-confrontation, dialogue

\section{AUTEUR}

\section{ANTOINE BONNEMAIN}

Université Clermont Auvergne, Laboratoire ACTé (EA 4281)

antoine.bonnemain@uca.fr 